\title{
Taenia crassiceps-Excreted/Secreted Products Induce a Defined MicroRNA Profile that Modulates Inflammatory Properties of Macrophages
}

\author{
Diana Martínez-Saucedo, ${ }^{1,2}$ Juan de Dios Ruíz-Rosado, ${ }^{2}$ César Terrazas, ${ }^{3}$ Blanca E. Callejas, ${ }^{1}$ \\ Abhay R. Satoskar, ${ }^{3}$ Santiago Partida-Sánchez, ${ }^{2}$ and Luis I. Terrazas $\left(\mathbb{D}^{1,4}\right.$ \\ ${ }^{1}$ Unidad de Biomedicina, Facultad de Estudios Superiores Iztacala, Universidad Nacional Autónoma de México, \\ Estado de México, Mexico \\ ${ }^{2}$ Center for Microbial Pathogenesis, The Research Institute at Nationwide Children's Hospital, Columbus, Ohio, USA \\ ${ }^{3}$ Department of Pathology, The Ohio State University, Columbus, OH, USA \\ ${ }^{4}$ Laboratorio Nacional en Salud, Facultad de Estudios Superiores Iztacala, Universidad Nacional Autónoma de México, \\ Estado de México, Mexico \\ Correspondence should be addressed to Luis I. Terrazas; literrazas@unam.mx
}

Received 10 January 2019; Accepted 26 March 2019; Published 14 May 2019

Guest Editor: Hadi M. Yassine

Copyright (C) 2019 Diana Martínez-Saucedo et al. This is an open access article distributed under the Creative Commons Attribution License, which permits unrestricted use, distribution, and reproduction in any medium, provided the original work is properly cited.

\begin{abstract}
Helminth parasites modulate immune responses in their host to prevent their elimination and to establish chronic infections. Our previous studies indicate that Taenia crassiceps-excreted/secreted antigens (TcES) downregulate inflammatory responses in rodent models of autoimmune diseases, by promoting the generation of alternatively activated-like macrophages (M2) in vivo. However, the molecular mechanisms triggered by TcES that modulate macrophage polarization and inflammatory response remain unclear. Here, we found that, while TcES reduced the production of inflammatory cytokines (IL-6, IL-12, and TNF $\alpha$ ), they increased the release of IL-10 in LPS-induced bone marrow-derived macrophages (BMDM). However, TcES alone or in combination with LPS or IL-4 failed to increase the production of the canonical M1 or M2 markers in BMDM. To further define the antiinflammatory effect of TcES in the response of LPS-stimulated macrophages, we performed transcriptomic array analyses of mRNA and microRNA to evaluate their levels. Although the addition of TcES to LPS-stimulated BMDM induced modest changes in the inflammatory mRNA profile, it induced the production of mRNAs associated with the activation of different receptors, phagocytosis, and M2-like phenotype. Moreover, we found that TcES induced upregulation of specific microRNAs, including miR-125a-5p, miR-762, and miR-484, which are predicted to target canonical inflammatory molecules and pathways in LPS-induced BMDM. These results suggest that TcES can modulate proinflammatory responses in macrophages by inducing regulatory posttranscriptional mechanisms and hence reduce detrimental outcomes in hosts running with inflammatory diseases.
\end{abstract}

\section{Introduction}

Helminth infections induce polarized $\mathrm{T}_{\mathrm{H}}$ 2-type biased immune responses that play a role in parasite expulsion, tissue repair, and regulation of unrelated inflammatory and autoimmune responses in the host [1-3]. The striking ability of helminth parasites in conferring protection from diseases of immune dysregulation has increased the attention into the immunomodulatory mechanisms evoked by these pathogens. Previous studies in our laboratory, using a murine model of cysticercosis, demonstrated that chronic infection with the helminth Taenia crassiceps or administration of its excreted/secreted products (TcES) ameliorates the development of experimental ulcerative colitis, autoimmune encephalomyelitis (EAE), and type 1 diabetes [4-8]. The ability of $T$. crassiceps and TcES to counteract these inflammatory 
responses was demonstrated to be dependent on a population of macrophages that produced markers of alternative activation (M2), such as PD-L2, IL-4R $\alpha$, MR, IL-10, ARG1, YM1, and FIZZ1 [9].

Macrophages can be activated towards an M2 phenotype after being stimulated with IL- 4 produced by $\mathrm{T}_{\mathrm{H}} 2$ lymphocytes during parasitic infections or exposure to allergens $[10,11]$. In contrast, released IFN- $\gamma$ and pathogen or danger-associated molecular patterns (PAMPs or DAMPs) during infections or tissue injury, respectively, promote classical (M1) activation in macrophages $[1,12]$. Although a crucial role for $T$. crassiceps-induced M2 macrophages in regulating detrimental autoimmune and inflammatory responses has been demonstrated [3], the transcriptional events elicited by TcES that modulate macrophage activation have not been elucidated.

Helminth infections and/or their antigens can trigger the levels of microRNAs to modulate inflammatory responses in the host [13-15]. MicroRNAs are small noncoding RNAs that regulate cell functions posttranscriptionally through direct binding to the $3^{\prime}$-UTR (untranslated region) of target messenger RNAs (mRNAs), resulting in the destabilization of mRNAs and repression of translation [16]. Recently, microRNAs have been associated with helminth-induced M2 macrophages in vitro and in vivo. For instance, Rückerl et al. reported that macrophages obtained during acute ( 3 weeks) Brugia malayi infection induced microRNAs associated with M2 macrophages, such as miR-199-5p, miR-378-3p, and miR-125b-5p [15]. In addition, Guo and Zheng identified distinct microRNAs, including miR-146a-5p, miR-155-5p, miR-21a-5p, miR-146b-5p, miR-99b-3p, miR-125a-5p, and miR-378, in RAW 264.7 macrophages cocultured with metacestodes of Echinococcus multilocularis. In these studies, the authors suggested a role for these microRNAs in targeting important inflammatory mRNAs (Tnf, Illa, Il6, Il12a, Ill2b, Ccl22, and Ccl18 mRNA) [14]. Thus, microRNAs may be a key mechanism elicited by helminths in the regulation of inflammatory responses in the host.

Although we have previously demonstrated a role for the TcES in preventing STAT1 phosphorylation in inflammatory macrophages [17], the influence of TcES in macrophage polarization and the transcriptional pathways regulating this process remain unknown. Here, we determined the effect of TcES alone or in combination with LPS or IL-4, in the regulation of multiple mRNA transcripts and microRNAs induced in macrophages. Our results indicate that TcES decreased the production of inflammatory cytokines (IL-12, $\mathrm{TNF} \alpha$, and IL-6) in LPS-induced macrophages but has a limited role in inducing directly the production of M1- and/or M2-associated molecules. The immune-modulatory ability of TcES was further associated with increased levels of specific microRNAs, which are predicted to target, according to our bioinformatic analysis, numerous inflammatory mRNAs involved in the TNF and NF- $\kappa \mathrm{B}$ signaling pathways. These findings suggest a role for TcES in modulating the transcriptional profile of macrophages via altering their microRNA profile and, consequently, the inflammatory properties of these immune cells.

\section{Materials and Methods}

2.1. Ethics Statement. All experiments in this study were performed according to the guidelines for the Care and Use of Laboratory Animals adopted by the US National Institutes of Health. The Institutional Animal Care and Use Committee (IACUC) at the Research Institute at Nationwide Children's Hospital and the Ohio State University approved all protocols.

2.2. Mice. Adult 6- to 8-week female $\mathrm{BALB} / \mathrm{c}$ mice were purchased from The Jackson Laboratory. All animals were maintained in a pathogen-free environment and established as breeding colonies in the Transgenic Mouse Facility at the Research Institute at Nationwide Children's Hospital or in specific pathogen-free conditions at the Ohio State University Laboratory Animal Resources. The mice were housed in sterilized polycarbonate cages with basic filter top caging containing pine wood shavings and were offered mouse ration and water ad libitum. The cages were held in Isolation and Containment cubicles (Britz and Co., Wheatland, WY).

2.3. Parasites and TcES. Metacestodes of T. crassiceps (ORF strain) were harvested under sterile conditions from the peritoneal cavity of female BALB/c mice after 8-10 weeks of intraperitoneal (i.p.) infection. The cysticerci were washed four times in physiological saline solution prior to maintaining them in culture with a sterile saline solution at $37^{\circ} \mathrm{C}$ for $24 \mathrm{~h}$. The supernatant was recovered and centrifuged for $10 \mathrm{~min}$ at $1000 \mathrm{~g}$. The heavy fraction of TcES was concentrated using the $50 \mathrm{kDa}$ Amicon Ultra Filter (Millipore), $30 \mathrm{~min}$ at $1000 \mathrm{~g}$. Protease inhibitors were added to the $>50 \mathrm{kDa}$ fraction, and samples were stored at $-70^{\circ} \mathrm{C}$ until further use.

2.4. Bone Marrow-Derived Macrophages (BMDM). To generate bone marrow-derived macrophages (BMDM), we followed the protocol previously described [18]. Briefly, bone marrow cells were obtained by flushing femurs and tibias from BALB/c mice with a sterile saline solution. The isolated cells were plated in $100 \mathrm{~mm}$ Petri dishes at $1 \times 10^{6}$ cells $/ \mathrm{mL}$ in Dulbecco's modified Eagle's media (DMEM, Mediatech, Herndon, VA), supplemented with $10 \%$ heat-inactivated fetal bovine serum (FBS) (Life Technologies, Grand Island, NY), 1\% penicillin/streptomycin, $1 \%$ glutamine, and $20 \mathrm{ng} / \mathrm{mL}$ of macrophage colonystimulating factor (M-CSF, BioLegend). On day 7, the cells were harvested, washed, counted, and replated in culture media (without M-CSF) at a density of $2 \times 10^{6}$ cells/well (12-well plate, Falcon polystyrene). BMDM were incubated with either TcES $(25 \mu \mathrm{g} / \mathrm{mL})$, Escherichia coli LPS (1 mg/mL, Sigma-Aldrich), interleukin-4 (20 ng/mL), TcES+LPS or TcES+IL-4. After 4 and $24 \mathrm{~h}$ poststimulation, BMDM were harvested for flow cytometric and transcriptomic analysis. The supernatants were recovered for cytokine detection by ELISA.

2.5. Flow Cytometric Analysis. Flow cytometric analysis was performed as previously described [19]. Briefly, harvested 
BMDM were incubated in $1 \mu \mathrm{g} / \mathrm{mL}$ of anti-mouse Fc receptor antibody in $100 \mathrm{~mL}$ PBS containing $0.5 \%$ BSA plus $0.02 \%$ $\mathrm{NaN}_{3}$ (FACS buffer) for $15 \mathrm{~min}$ on ice. Subsequently, single-cell suspensions were stained for $15 \mathrm{~min}$ at $4^{\circ} \mathrm{C}$ with blue-fluorescent reactive dye, L23105 (Life Technologies) to discriminate dead cells. After washing, 1-3 $\times 10^{6}$ cells were surface-stained in FACS buffer for $15 \mathrm{~min}$ at $4^{\circ} \mathrm{C}$ with antibodies recognizing CD11b (Alexa Fluor 700, BioLegend), F4/80 (Brilliant Violet 785, BioLegend), CD86 (Brilliant Violet 421, BioLegend), PD-L1 (PE-Cy7, BioLegend), and PD-L2 (PE, BioLegend). Surface-stained cells were washed three times with FACS buffer and treated with Fix/Perm reagent according to the protocol of the cytofix/cytoperm kit (BD Biosciences, San Jose, CA, USA). The cells were intracellularly stained in FACS buffer containing anti-Nos2 (PE, eBiosciences) and anti- $\mathrm{h} / \mathrm{m}$ arginase 1 (APC, R\&D systems) for $30 \mathrm{~min}$ at $4^{\circ} \mathrm{C}$ and further collected on an LSR II cytofluorometer (BD, Franklin Lakes, NJ). Stained cells were gated according to size (SSC-A) and forward scatter (FSC-A) to eliminate debris. Doublets were excluded from the analysis by using forward scatter height (FSC-H) and FSC-A. Data analysis was performed using FlowJo Software (FlowJo, LLC).

2.6. Cytokine Assay. Supernatants from cell cultures of stimulated macrophages were recovered at 4 and $24 \mathrm{~h}$ poststimulation, and the levels of the cytokines IL-10, IL-6, TNF $\alpha$, and IL-12 were measured by ELISA according to the manufacturer's instructions (PeproTech).

2.7. RNA Extraction and Arrays. Total RNA was extracted from BMDM stimulated with LPS $\left(\mathrm{M}_{\mathrm{LPS}}\right)$, TcES $\left(\mathrm{M}_{\mathrm{TcES}}\right)$, LPS+TcES $\left(\mathrm{M}_{\mathrm{TcES}+\mathrm{LPS}}\right)$, or culture media $\left(\mathrm{M}_{0}\right)$ using QIAzol reagent (QIAGEN), according to the manufacturer's specifications, and stored at $-80^{\circ} \mathrm{C}$. Next, RNA was purified following the miRNeasy kit protocol (QIAGEN). RNA concentration and integrity were determined using a NanoDrop $^{\mathrm{TM}}$ spectrophotometer (Thermo Scientific, Wilmington, DE) and Agilent Bioanalyzer 2100, respectively. For transcriptomic analysis, $50 \mathrm{ng} / \mu \mathrm{L}$ of RNA was used for the nCounter Inflammation Panel (NanoString mRNAs) and the nCounter miRNA Assay set (microRNAs). Both mRNA and microRNA arrays were performed following the manufacturer's instructions at the Genomics Shared Resource, OSU. Data analysis for the nCounter Inflammatory Panel (mRNA) and for the nCounter miRNA Assay set was conducted using the nSolver ${ }^{\mathrm{TM}}$ Analysis Software according to the manufacturer. For the nCounter Inflammatory Panel (mRNA), we normalized using the normalization factor and subtracted the background (mean of negative controls \pm 2 standard deviations). Next, we normalized using the geometric mean of housekeeping genes as reported [20]. Then, using the normalized counts, we calculated the fold change (FC) by comparing $\mathrm{M}_{\mathrm{TcES}}, \mathrm{M}_{\mathrm{TcES}+\mathrm{LPS}}$, and $\mathrm{M}_{\mathrm{LPS}}$ to $\mathrm{M}_{0}$. For the nCounter miRNA Assay set, we first normalized using the normalization factor. The background was subtracted from the data using the mean of negative controls \pm 2 standard deviations. Finally, we used the top 75 microRNAs [21]. The normalized counts were used to calculate the FC by comparing $\mathrm{M}_{\mathrm{TcES}}, \mathrm{M}_{\mathrm{TcES}+\mathrm{LPS}}$, and $\mathrm{M}_{\mathrm{LPS}}$ to $\mathrm{M}_{0}$. Of the 566 total probes measured in the assay, 183 and 236 microRNAs for $4 \mathrm{~h}$ and $24 \mathrm{~h}$, respectively, were identified and used for analyzing significant changes in microRNA levels among samples. MultiExperiment Viewer $(\mathrm{MeV})$ was used to generate heat maps, which represent $\log _{2}$-transformed data.

2.8. Real-Time PCR. TaqMan gene expression assays (Applied Biosystems) were used to quantify and/or validate the levels of mRNAs and microRNA transcripts. cDNA was generated from mRNAs, using a $15 \mu \mathrm{L}$ RT reaction consisting of $2.0 \mu \mathrm{L}$ of Buffer (10x), $0.8 \mu \mathrm{L} 100 \mathrm{mM}$ dNTPs (100 mM), $1.0 \mu \mathrm{L}$ reverse transcriptase, $2.0 \mu \mathrm{L}$ of mRNA primer, and $1 \mu \mathrm{g}$ of total RNA. RT reaction was incubated for $30 \mathrm{~min}$ at $16^{\circ} \mathrm{C}, 30 \mathrm{~min}$ at $42^{\circ} \mathrm{C}$, and $5 \mathrm{~min}$ at $85^{\circ} \mathrm{C}$. For microRNA levels, a $15 \mu \mathrm{L}$ reaction was prepared with $2.0 \mu \mathrm{L}$ of buffer (10x), $0.2 \mu \mathrm{L} 100 \mathrm{mM}$ dNTPs $(100 \mathrm{mM}), 1.0 \mu \mathrm{L}$ reverse transcriptase, $0.2 \mu \mathrm{L}$ RNAse inhibitor $(20 \mathrm{U} / \mu \mathrm{L}), 3.0 \mu \mathrm{L}$ of microRNA primer, and $100 \mathrm{ng}$ of total RNA. RT reaction was incubated as mentioned before. For both mRNA and microRNAs, quadruplicate real-time PCR reactions were performed in the 7500 Real-Time PCR system. The amplification reaction mix was composed of $10 \mu \mathrm{L}$ of TaqMan Universal PCR Master Mix (2x), $1 \mu \mathrm{L}$ of the specific mRNA or microRNA probe, and $1 \mu \mathrm{L}$ of specific microRNA cDNA. The reactions were preincubated for 10 minutes at $95^{\circ} \mathrm{C}$ and amplified with 40 cycles consisting of $10 \mathrm{sec}$ at $95^{\circ} \mathrm{C}$, $40 \mathrm{sec}$ at $60^{\circ} \mathrm{C}$, and $5 \mathrm{sec}$ at $72^{\circ} \mathrm{C}$ (fluorescence acquisition). To assess possible bias for reference RNA, we used $18 \mathrm{~S}$ RNA, Actb, and Gapdh mRNAs. Relative quantification was calculated by $2^{-\Delta \Delta \mathrm{Ct}}$. All mRNA and microRNA assays were tested for reproducibility and linearity (PCR efficiency was between 1.9 and 2.0 for all assays). All primers were purchased from Applied Biosystems. The primer sequences are shown in Table S1.

2.9. $m R N A$ and MicroRNA Target Gene Prediction and Bioinformatics Analysis. Target mRNAs of differentially produced microRNAs were predicted using DIANATarBase database v6.0, which includes experimentally validated targets from the literature. To explore the potential biological function of the microRNAs' profile and their targets, DIANA-mirPath v2.0 (http://snf-515788.vm.okeanos.grnet .gr/) was used to perform enrichment analysis of microRNA's target mRNAs in the KEGG pathway and in GO terms [22].

2.10. Statistical Analysis. Data analyses were performed using GraphPad Prism 6 software. Statistical comparisons were performed by using Student's $t$-test. $p$ values less than 0.05 were considered significant. Graphed data are presented as mean \pm SD or SEM.

\section{Results}

3.1. TcES Reduces the Inflammatory Response of LPS-Induced $B M D M$. Previously, we demonstrated the ability of TcES in reducing the development of inflammatory and autoimmune diseases in rodent models [4-8]. The effect of TcES in 
counteracting detrimental inflammatory responses in vivo is associated with the emergence of polarized macrophages towards an M2 phenotype $[4,5,11]$. Although studies in our laboratory indicate a role for TcES in blocking the IFN$\gamma /$ STAT1 signaling pathway in macrophages [17], the effect of TcES in inducing directly M2 macrophages remains to be elucidated. To define the macrophage profile elicited by TcES, we first determined the levels of the inflammatory cytokines IL-12, IL-6, TNF $\alpha$, and IL-10 in cultures from BMDM. The cells were stimulated (Figure 1(a)) for $4 \mathrm{~h}$ or $24 \mathrm{~h}$ with TcES (henceforth $\mathrm{M}_{\mathrm{TcES}}$ ), E. coli lipopolysaccharide $\left(\mathrm{M}_{\mathrm{LPS}}\right)$, interleukin-4 $\left(\mathrm{M}_{\mathrm{IL}-4}\right)$, TcES+LPS $\left(\mathrm{M}_{\mathrm{TcES}+\mathrm{LPS}}\right)$, TcES+IL-4 $\left(\mathrm{M}_{\mathrm{TcES}+\mathrm{IL}-4}\right)$, or PBS $\left(\mathrm{M}_{0}\right)$. Supernatants obtained from $\mathrm{M}_{\mathrm{TcES}}$ displayed higher levels of IL-10 and deficient levels of inflammatory cytokines (IL-6 and $\mathrm{TNF} \alpha$ ) compared to all the groups at $4 \mathrm{~h}$ poststimulus (Figures 1(b) and 1(e)). However, IL-10 production by $\mathrm{M}_{\mathrm{TcES}}$ did not continue at $24 \mathrm{~h}$. Interestingly, we found that exposure of macrophages to TcES and stimulated with LPS ( $\left.\mathrm{M}_{\mathrm{TcES}+\mathrm{LPS}}\right)$ significantly reduced the production of IL-12, IL-6, and TNF $\alpha$ compared to those in $M_{L P S}$ at $24 \mathrm{~h}$ (Figures 1(c) and 1(e)). Increased IL-10 levels were observed in supernatants from $\mathrm{M}_{\mathrm{TcES}+\mathrm{LPS}}$ compared with all groups at $24 \mathrm{~h}$ (Figure $1(\mathrm{~b})$ ). A similar trend was identified in the levels of the mRNA for Tnf at $24 \mathrm{~h}$ poststimulus (Figure 1(f)), whereas levels of Il10 mRNA were similar between all groups at $24 \mathrm{~h}$ (Figure $1(\mathrm{~g})$ ). Our results suggest that TcES play a role in downregulating the production of proinflammatory cytokines in LPS-induced $\mathrm{BMDM}$, by increasing the production of a regulatory cytokine.

To gain insight in the phenotypic profile induced by TcES in macrophages, we used flow cytometry technique to determine the production of intracellular nitric oxide synthase (NOS2), and arginase-1 (ARG1), as the conventional markers for M1 and M2 profiles, respectively, in BMDM. Our results showed that while $\mathrm{M}_{\mathrm{LPS}}$ and $\mathrm{M}_{\mathrm{IL}-4}$ presented increased percentages of $\mathrm{NOS}^{+}$and $\mathrm{ARG}^{+}$macrophages, respectively, $\mathrm{M}_{\mathrm{TcES}}$ displayed limited production of these molecules (Figures 2(a) and 2(d)). Additionally, similar percentages of $\mathrm{NOS}^{+}$BMDM were found between $\mathrm{M}_{\mathrm{TcES}+\mathrm{LPS}}$ and $\mathrm{M}_{\mathrm{LPS}}$, and comparable $\mathrm{ARG} 1^{+} \mathrm{BMDM}$ were observed when analyzing $\mathrm{M}_{\mathrm{TcES}+\mathrm{IL}-4}$ versus $\mathrm{M}_{\mathrm{IL}-4}$ (Figures 2(a) and 2(d)). Levels of mRNA Arg1 by RT-qPCR showed similar trends as the flow cytometric analysis (Figure 2(f)). While the levels of Nos2 mRNA were upregulated in $\mathrm{M}_{\text {TcES+LPS }}$ compared to $M_{0}$ but significantly reduced compared to $M_{L P S}$ (Figure 2(e)). These data suggest that the stimulus with TcES, either alone or in combination with LPS or IL-4, has a limited role in inducing the production of canonical M1 or M2 markers. Nevertheless, these antigens play a role in downregulating the proinflammatory response to LPS in BMDM.

3.2. TcES Modify the Proinflammatory mRNA Profile of LPSInduced BMDM. Because our data suggest a novel role for TcES in attenuating the proinflammatory response of LPSinduced BMDM, and the current M1/M2 paradigm scarcely describes the influence of TcES in the transcriptional profile of macrophages, we performed a proinflammatory mRNA array screen (see "mRNA array" for details) on $\mathrm{M}_{0}, \mathrm{M}_{\mathrm{LPS}}$, $\mathrm{M}_{\mathrm{TcES}}$, and $\mathrm{M}_{\mathrm{TcES}+\mathrm{LPS}}$, at 4 and $24 \mathrm{~h}$ poststimulus (Figure 3). Commonly produced mRNAs among the groups of $\mathrm{M}_{\mathrm{LPS}}, \mathrm{M}_{\mathrm{TcES}}$, and $\mathrm{M}_{\mathrm{TcES}+\mathrm{LPS}}$ are displayed in Table S2. As expected, our results indicate increased levels of multiple proinflammatory mRNAs in $M_{\text {LPS }}$ with respect to $M_{0}$ (Table 1 and Table S3), including Illa, Il6, Il12a, Il12b, Tnf, and Nos2, among other mRNAs, at 4 and $24 \mathrm{~h}$ poststimulus. These molecules correspond to previously reported markers for LPS-stimulated macrophages [1]. In contrast, $\mathrm{M}_{\mathrm{TcES}}$ downregulated the levels, with respect to $\mathrm{M}_{0}$, of cytokines, chemokines, and transcriptional factors distinctive of M1-activated macrophages, while displaying upregulated levels mainly associated with enzymes, as MAPK pathway, at 4 and $24 \mathrm{~h}$ poststimulus (Table 1 and Table S4). Noticeably, although $\mathrm{M}_{\text {TcES+LPS }}$ presented 132 and 96 upregulated mRNAs (Table S5), these macrophages only shared 6 and 3 upregulated mRNAs with $M_{\text {TcES }}$ at 4 and $24 \mathrm{~h}$ poststimulus, respectively. However, $\mathrm{M}_{\mathrm{TcES}+\mathrm{LPS}}$ shared 89 and 65 upregulated mRNAs with $M_{\text {LPS }}$ at 4 and $24 \mathrm{~h}$ poststimulus, respectively, including transcripts for cytokines, chemokines, receptors, and transcriptional factors as Illa, Illb, Il6, Il12a, Il12b, Ccl3, Ccl5, Ccl2, Ccl7, Cd86, Tlr2, Stat1, Stat3, and Nfkb1 mRNA. The differentially induced mRNAs between $M_{\text {TcES+LPS }}$ and $M_{L P S}$ are shown in Table S6. Next, we validated 7 mRNAs associated with M1 (Il1b, Stat1, Cd86, Il6, and Il12b) and M2 (Stat6 and Chi3l3) macrophages by RT-qPCR. The levels of these mRNAs were comparable to those observed in the mRNA array (Figure 4), which attest for the high quality of our array, supporting that a posttranscriptional mechanism induced by TcES may have a role in macrophage's response to LPS. Interestingly, although the levels of IL- 6 and IL-12 in supernatants from $M_{\mathrm{TcES}+\mathrm{LPS}}$ were significantly reduced respect to $M_{\text {LPS }}$ (Figures $1(a)$ and 1(b)), the levels of their mRNAs of these cytokines were comparable between $\mathrm{M}_{\mathrm{TCES}+\mathrm{LPS}}$ and $\mathrm{M}_{\mathrm{LPS}}$. These data suggest that posttranscriptional mechanisms triggered by TcES may have a role in modulating the production of specific inflammatory cytokines.

3.3. TcES Modulate the Profile of MicroRNAs in LPSStimulated BMDM. MicroRNAs participate in diverse biological processes at the posttranscriptional regulatory level. The complementary binding of microRNAs to mRNAs reduces either transcription or translation of mRNA transcripts [16]. Recently, a handful of studies indicate a role for helminth parasites and their antigens in inducing microRNAs to modulate host immune responses [14, 15, 23]. To determine whether the ability of TcES in attenuating the inflammatory response of BMDM is associated with the production of specific microRNAs, we performed a microRNA array (see "microRNA array" in Materials and Methods for details) in $\mathrm{M}_{0}, \mathrm{M}_{\mathrm{LPS}}, \mathrm{M}_{\mathrm{TcES}}$, and $\mathrm{M}_{\mathrm{TcES}+\mathrm{LPS}}$, at 4 and $24 \mathrm{~h}$ poststimulus. As a result, we identified 7 and 89 upregulated microRNAs in $\mathrm{M}_{\mathrm{LPS}}$ at $4 \mathrm{~h}$ and $24 \mathrm{~h}$, respectively. $\mathrm{M}_{\mathrm{TcES}}$ displayed $13(4 \mathrm{~h})$ and $3(24 \mathrm{~h})$, and $\mathrm{M}_{\text {TcES+LPS }}$ showed 19 (4h) and $28(24 \mathrm{~h})$ upregulated microRNAs (Figure 5). The top 10 up- and downregulated microRNAs in $\mathrm{M}_{\mathrm{LPS}}, \mathrm{M}_{\mathrm{TcES}}$, and 


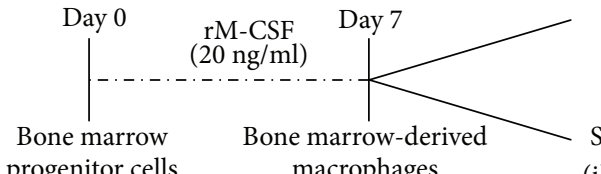
macrophages (BMM)

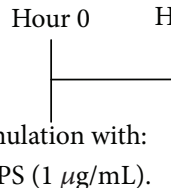

(i) LPS $(1 \mu \mathrm{g} / \mathrm{mL})$.

(ii) IL-4 $(20 \mathrm{ng} / \mathrm{mL})$.

(iii) $\operatorname{TcES}(25 \mu \mathrm{g} / \mathrm{mL})$.

(iv) TcES+LPS

$(25 \mu \mathrm{g} / \mathrm{mL}+1 \mu \mathrm{g} / \mathrm{mL})$
Hour 4

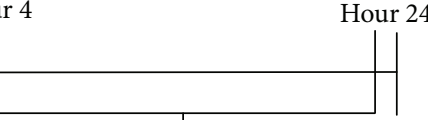

(i) Retrieval of supernatant, RNA and cells.

(ii) Flow cytometry.

(iii) Cytokine detection in supernatant.

(iv) mRNA and microRNA array.

(v) RT-qPCR.

(a)

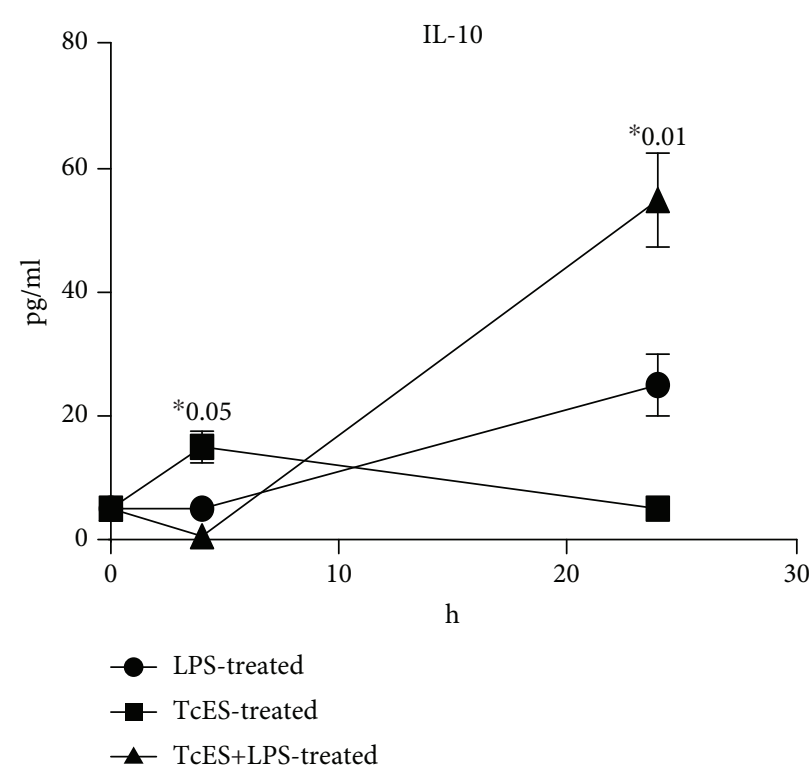

(b)

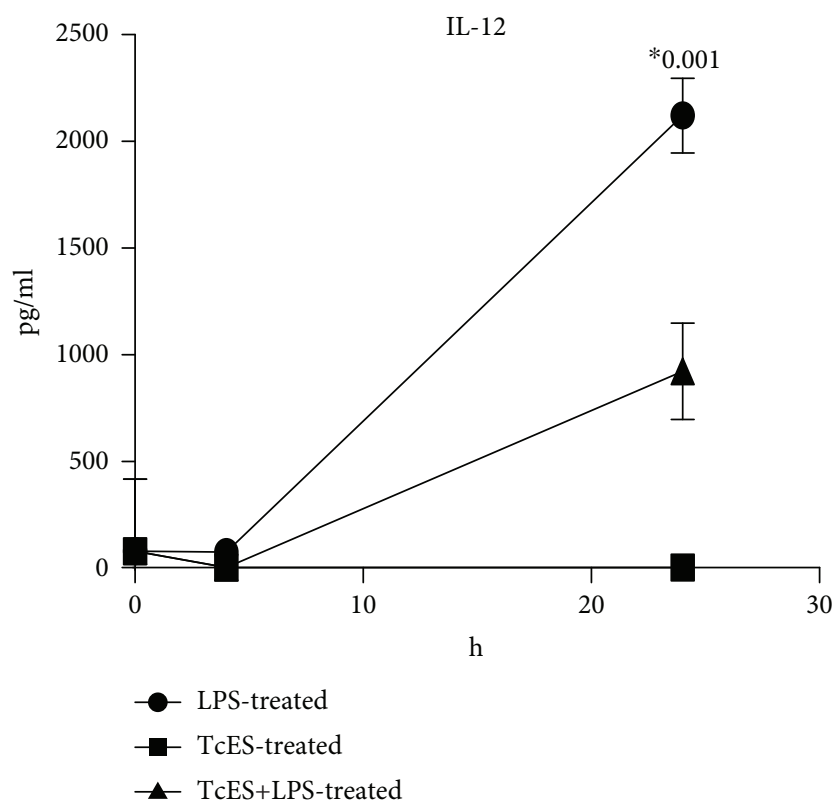

(d)

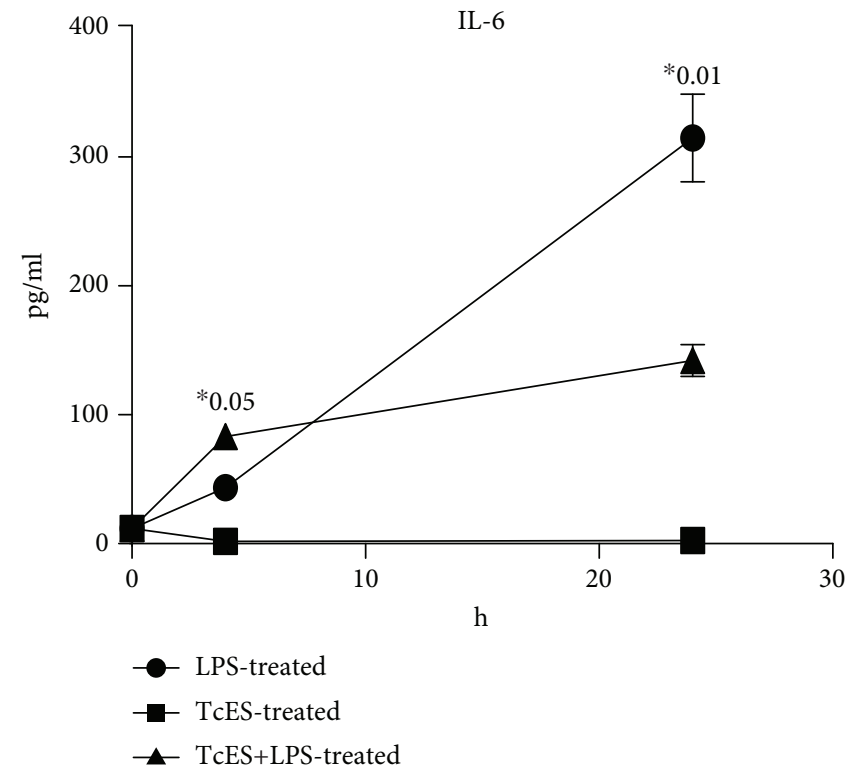

(c)

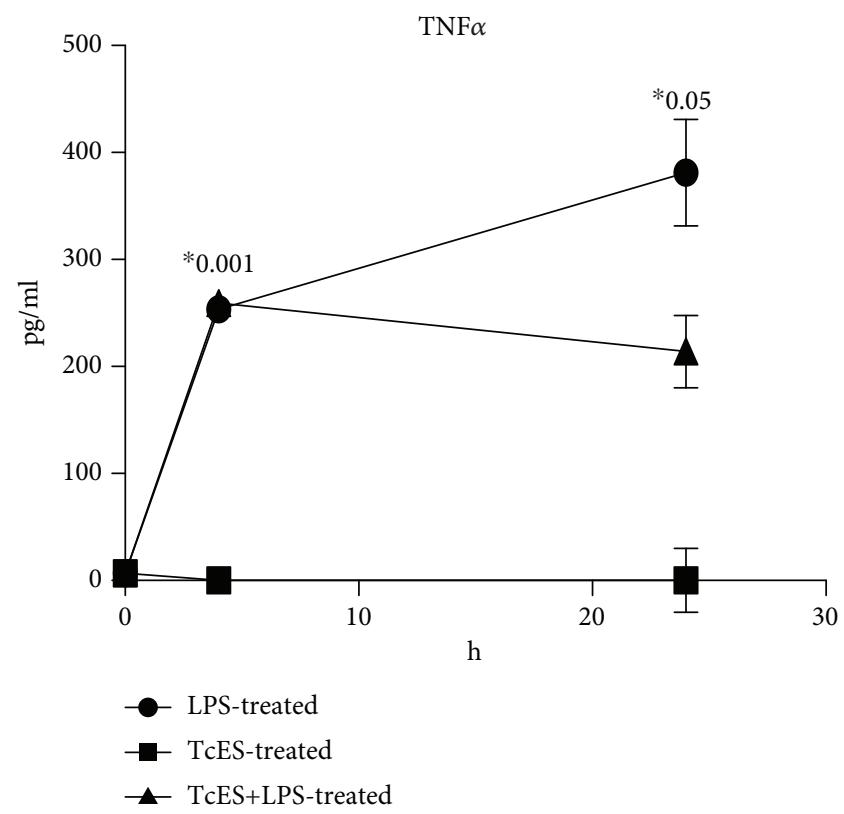

(e)

Figure 1: Continued. 


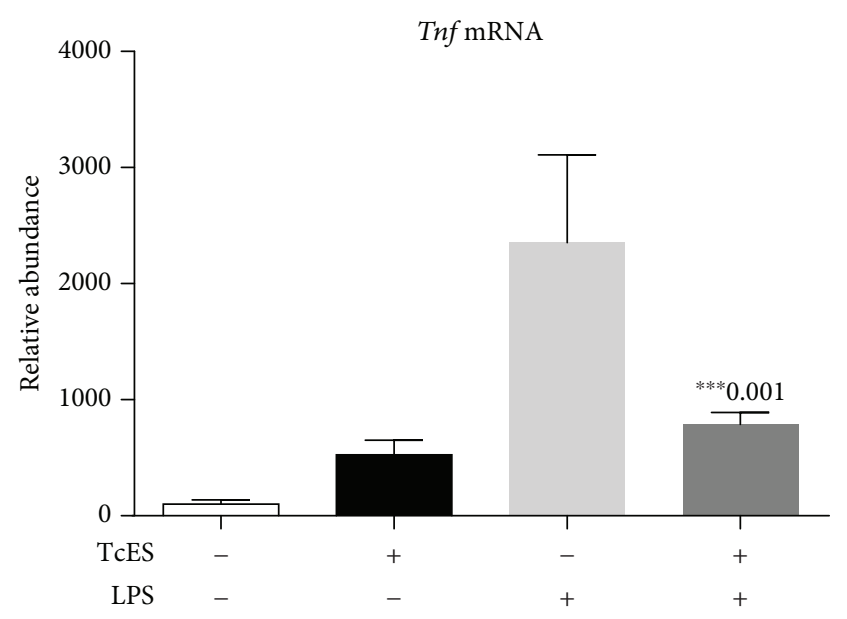

(f)

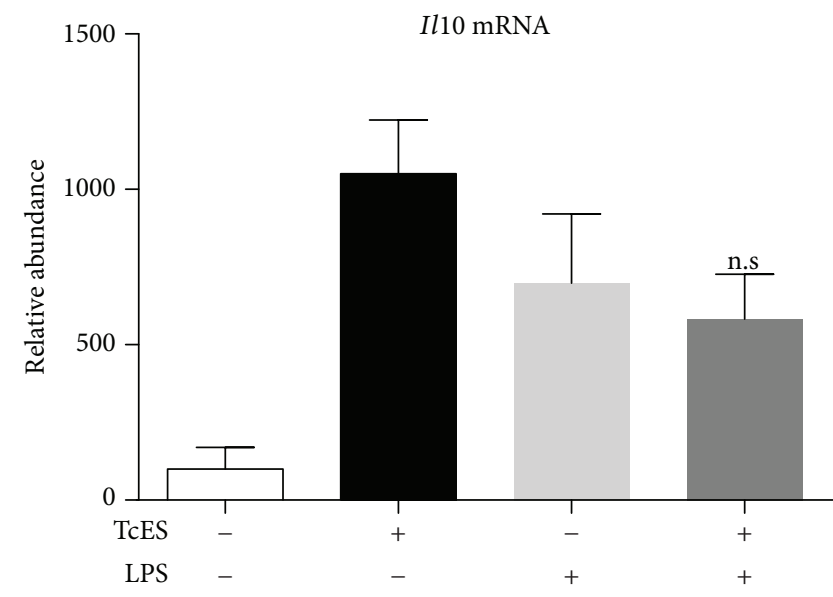

(g)

FIGURE 1: TcES regulates the production of inflammatory cytokines in LPS-induced macrophages. (a) Flow diagram of general experimental design. For differentiation of macrophages, bone marrow progenitor cells were cultured with rM-CSF at $37^{\circ} \mathrm{C} 5 \% \mathrm{CO}_{2}$ for 7 days. On completion day, BMDM were washed and stimulated with the following stimuli: LPS $(1 \mu \mathrm{g} / \mathrm{mL})$, TcES $(25 \mathrm{ng} / \mathrm{mL})$, or both for 4 or 24 hours. The supernatants, total RNA or cells were harvested for later procedures. (b) Kinetics levels of IL-10, (c) IL-6, (d) IL-12, and (e) TNF $\alpha$ in supernatants from stimulated BMDM. Evaluation of the levels of (f) Tnf and (g) Illo mRNA by RT-qPCR in groups of stimulated macrophages mentioned above $(n=6,3$ replicates condition). mRNA levels are represented as mean relative $( \pm S D)$. Data are representative of 3 independent experiments using 3 replicates per stimuli. Significance was calculated using $t$-test. ${ }^{*} p<0.01,{ }^{* *} p<0.05$, and ${ }^{* * *} p<0.001$.

$\mathrm{M}_{\mathrm{TcES}+\mathrm{LPS}}$ are shown in Table 2 . The complete lists of microRNAs are shown in Table S7-S9. Additionally, we found 4 and 2 microRNAs shared among the groups of stimulated BMDM at 4 and $24 \mathrm{~h}$, respectively (Table S10). Interestingly, $\mathrm{M}_{\mathrm{TcES}+\mathrm{LPS}}$ shared 6 upregulated microRNAs with $\mathrm{M}_{\mathrm{TCES}}$ and only 3 with $\mathrm{M}_{\mathrm{LPS}}$ at $4 \mathrm{~h}$ poststimulus. However, $M_{\text {TcES+LPS }}$ did not share microRNAs with $\mathrm{M}_{\mathrm{TcES}}$ and shared 22 with $\mathrm{M}_{\text {LPS }}$ at $24 \mathrm{~h}$ poststimulus. Finally, $\mathrm{M}_{\text {TcES+LPS }}$ differentially induced 3 and 20 microRNAs compared to $\mathrm{M}_{\mathrm{LPS}}$ at 4 and $24 \mathrm{~h}$ poststimulation, respectively (Table S11). These data suggest that TcES induce the early production $(4 \mathrm{~h})$ of microRNAs, followed by the stimulus with LPS $(24 \mathrm{~h})$, in $\mathrm{M}_{\mathrm{TcES}+\mathrm{LPS}}$. This phenomenon is associated with an increased number of upregulated microRNAs in $\mathrm{M}_{\mathrm{TcES}}$ compared to $\mathrm{M}_{\mathrm{LPS}}$ (13 vs. 7 microRNAs, Figure 5) at $4 \mathrm{~h}$ poststimulation.

To assess the potential biological relevance of the upregulated microRNAs in the transcriptional profile of activated macrophages, we conducted bioinformatic analysis as GO terms and KEGG pathway analysis by comparing $\mathrm{M}_{\mathrm{TcES}}$, $\mathrm{M}_{\text {TcES+LPS }}$, or $\mathrm{M}_{\mathrm{LPS}}$ vs. $\mathrm{M}_{0}$ at both 4 and $24 \mathrm{~h}$ poststimulus. The GO terms in $\mathrm{M}_{\mathrm{TcES}}$ and $\mathrm{M}_{\mathrm{TCES}+\mathrm{LPS}}$ were mainly enriched for the biological process associated with anatomical structure development, cell differentiation, and cellular protein differentiation process at $4 \mathrm{~h}$ poststimulus (Figures 6(a) and 6(b)). Anatomical structure development, cell differentiation, and chromosome organization were predicted to be a target by microRNAs in $\mathrm{M}_{\mathrm{TcES}}$, while organelle, anatomical structure, and cell differentiation were enriched in $\mathrm{M}_{\mathrm{TcES}+\mathrm{LPS}}$ at $24 \mathrm{~h}$ poststimulus (Figures $6(\mathrm{~d})$ and $6(\mathrm{e})$ ). Lastly, GO terms enriched for $\mathrm{M}_{\mathrm{LPS}}$ are chromosome organization, biosynthetic process, and protein complex as well as organelle, anatomical structure, and cell differentiation at 4 and $24 \mathrm{~h}$ poststimulus, respectively (Figures 6(c) and 6(f)). The KEGG pathway enrichment analysis revealed that at $4 \mathrm{~h}$ stimulus, upregulated microRNAs were regulating glioma, chronic myeloid leukemia, and TGF- $\beta$ signaling pathway in $\mathrm{M}_{\mathrm{TcES}}$ (Figure 6(a)); ubiquitin-mediated proteolysis, p53, and GhRH signaling pathway in $\mathrm{M}_{\mathrm{TcES}+\mathrm{LPS}}$ (Figure $6(\mathrm{~b})$ ); and prostate cancer, steroid biosynthesis, and FoxO signaling pathway in $\mathrm{M}_{\text {LPS }}$ (Figure 6(c)). In contrast, the KEGG enrichment pathways at $24 \mathrm{~h}$ poststimulus were axon guidance, insulin signaling pathway, and HTLV-I infection in $\mathrm{M}_{\mathrm{TcES}}$ +LPS (Figure 6(e)) and inositol phosphate metabolism, pathways in cancer, and insulin signaling pathway in $M_{L P S}$ (Figure 6(f)). For more details of GO enrichment analysis and KEGG pathways, refer to Table S12 and Table S13. These data suggest a role for TcES in inducing microRNAs that regulate important metabolic, cell signaling, and inflammatory pathways in LPS-stimulated BMDM.

Next, we selected and validated by RT-qPCR four microRNAs (miR-125a-5p, miR-762, miR-155-5p, and miR-484), which are potentially involved in the regulation of inflammatory mRNAs, as indicated by previous studies and our bioinformatics analysis. We found that both $M_{\text {LPS }}$ and $M_{\text {TCES }}$ showed increased levels of miR-125a-5p (Figure 7(a)), a microRNA reported to reduce the production of inflammatory cytokines (IL-6, IL-12, and TNF $\alpha$ ) [24]. The levels of miR-125a-5p were sustained in $\mathrm{M}_{\mathrm{LPS}}$ and $\mathrm{M}_{\mathrm{TcES}+\mathrm{LPS}}$ until $24 \mathrm{~h}$ poststimulus (Figure $7(\mathrm{~b})$ ). The combined stimuli of TcES and LPS induced an additive effect in the levels of this microRNA at $4 \mathrm{~h}$ poststimulation (Figure $7(\mathrm{a})$ ). Furthermore, $\mathrm{M}_{\mathrm{TcES}}$ and $\mathrm{M}_{\mathrm{TcES}+\mathrm{LPS}}$ showed increased levels of miR-762, a microRNA known to directly target the mRNA of 

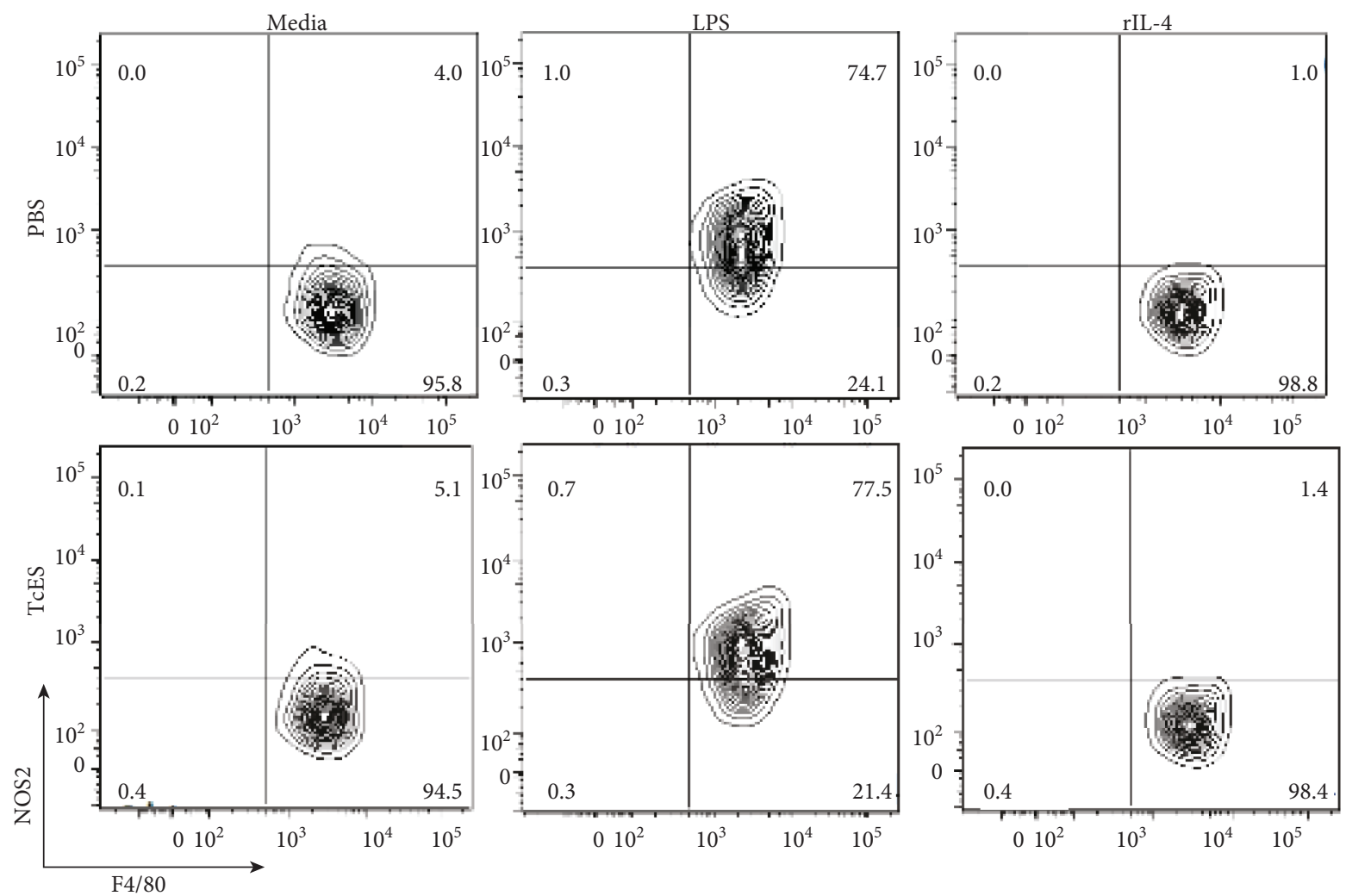

(a)
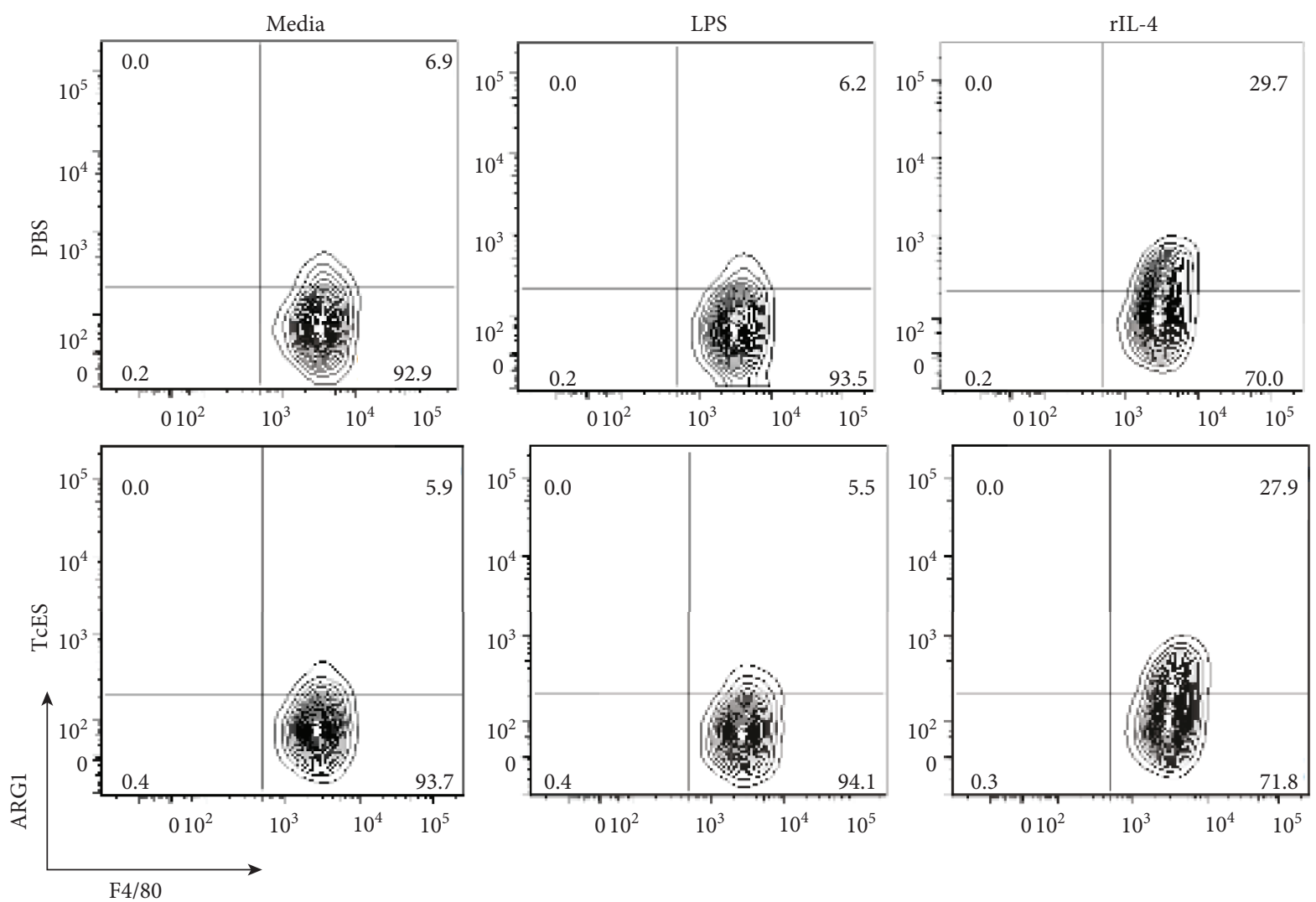

(b)

Figure 2: Continued. 


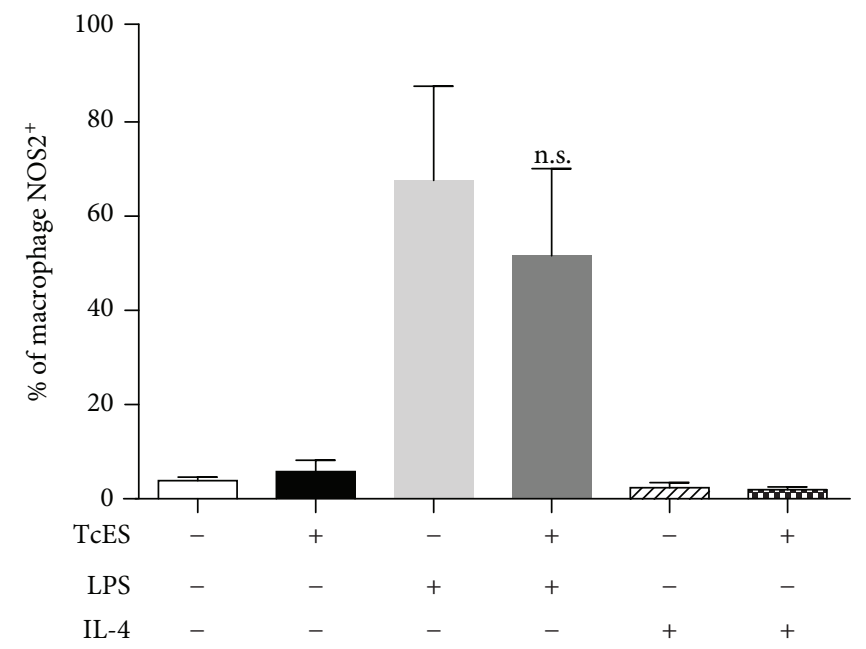

(c)

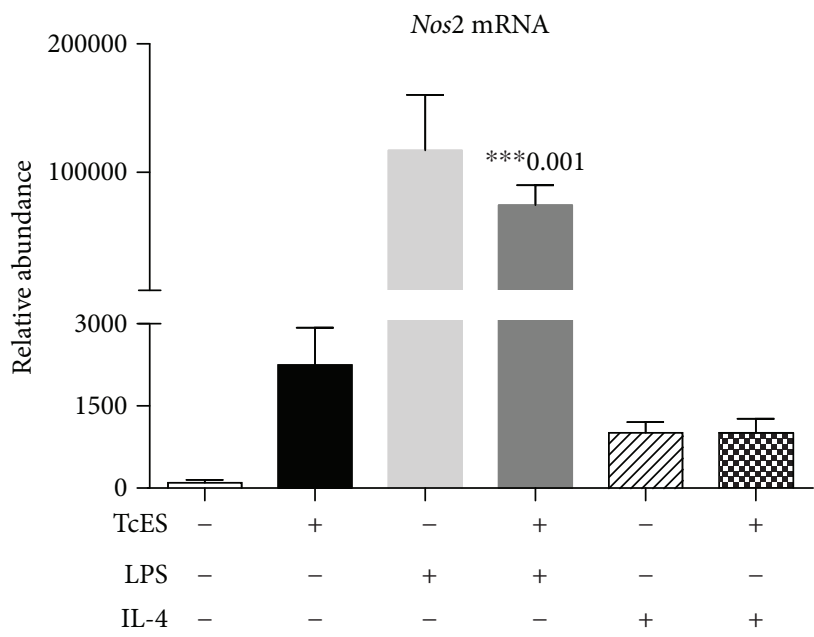

(e)

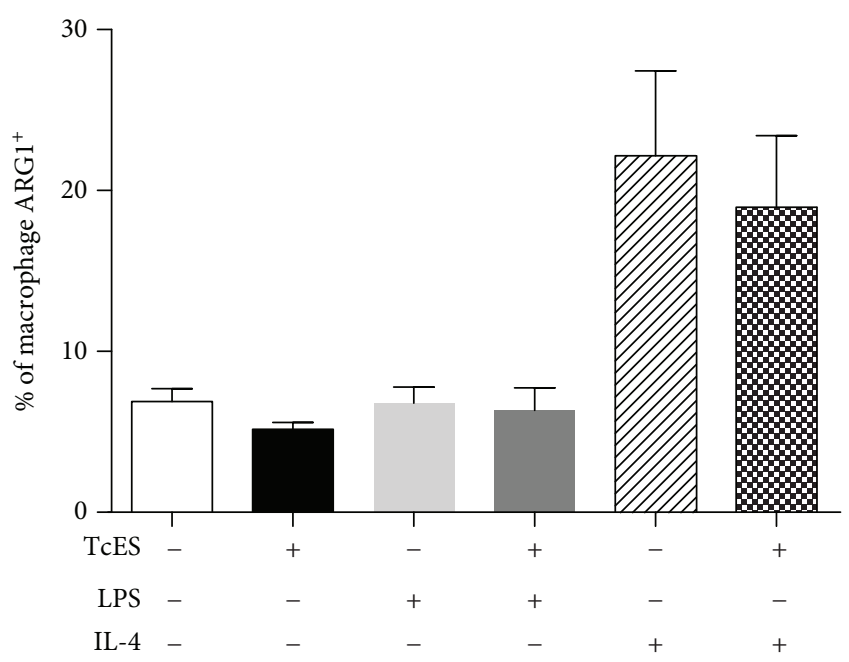

(d)

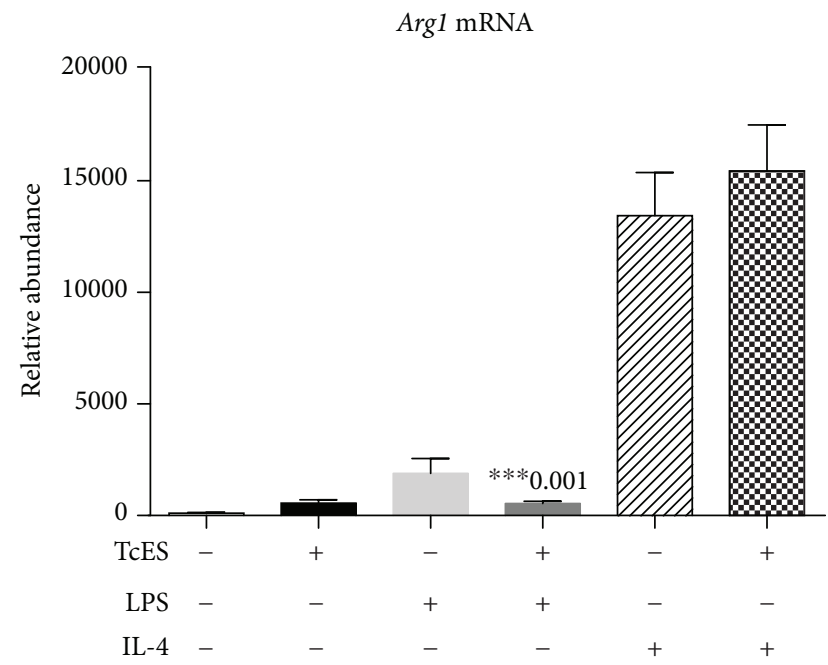

(f)

FIgURE 2: TcES do not modify the production of canonical M1/M2 macrophage markers. Representative dot plots, obtained by flow cytometry, of (a) $\mathrm{F} 4 / 80^{+} \mathrm{NOS}_{2}{ }^{+}$and (b) $\mathrm{F} 4 / 80^{+} \mathrm{ARG} 1^{+} \mathrm{BMDM}$, after $24 \mathrm{~h}$ poststimulus with one of the following stimuli: LPS (1 $\mu \mathrm{g} / \mathrm{mL}$ ), TcES $(25 \mathrm{ng} / \mathrm{mL}), \mathrm{IL}-4(20 \mathrm{ng} / \mathrm{mL})$, TcES+LPS, TcES+IL-4, or PBS. Bar graphs representing the percentage of (c) F4/80 ${ }^{+} \mathrm{NOS} 2^{+}$and $(\mathrm{d})$ F4 $/ 80^{+} \mathrm{ARG} 1^{+}$BMDM at $24 \mathrm{~h}$ poststimulus. (e) Evaluation of the levels of Nos2 and (f) Arg1 mRNA by RT-qPCR in BMDM stimulated for $24 \mathrm{~h}(n=6,3$ replicates condition). mRNA levels are represented as mean relative $( \pm \mathrm{SD})$. Data are shown as a representative of two independent experiments. Significance was calculated using $t$-test. ${ }^{*} p<0.01,{ }^{* *} p<0.05$, and ${ }^{* * *} p<0.001$.

inflammatory transcription factor Irf7 [25], at $4 \mathrm{~h}$ poststimulus (Figure $7(\mathrm{c})$ ). This microRNA was later produced in $\mathrm{M}_{\text {LPS }}$ at $24 \mathrm{~h}$ poststimulation (Figure $7(\mathrm{~d})$ ). In addition, miR-484 was highly produced in $\mathrm{M}_{\mathrm{TcES}}$ compared to both $\mathrm{M}_{\mathrm{TcES}+\mathrm{LPS}}$ and $\mathrm{M}_{\mathrm{LPS}}$ at 4 and $24 \mathrm{~h}$ poststimulus (Figures $7(\mathrm{e})-7(\mathrm{f})$ ). Our bioinformatic analysis suggests that miR-484 can potentially target Nfkb, Stat5a, Irf1, Myd88, Stat1, and Il12a mRNAs. Finally, miR-155-5p, a well-defined microRNA in M1 macrophages, was upregulated in $\mathrm{M}_{\mathrm{LPS}}$ and $\mathrm{M}_{\mathrm{TCES}+\mathrm{PS}}$ compared to $\mathrm{M}_{\mathrm{TCES}}$ at 4 and $24 \mathrm{~h}$ poststimulation (Figures $7(\mathrm{~g})-7(\mathrm{~h}))$. The profile of these miRNAs was comparable to those observed in the microRNA array. Altogether, our findings suggest a role for miR-125a-5p, miR762 , and miR-484 in the immunomodulatory effect of TcES in BMDM.

\section{Discussion}

Helminth parasites and their antigens can counteract proinflammatory responses generated during autoimmune diseases [3]. In our laboratory, we have previously demonstrated that infection with the helminth parasite T. crassiceps or the administration of TcES reduced the symptoms of EAE, type I diabetes, and ulcerative colitis, in part due to the polarization of macrophages in vivo towards an M2 phenotype $[4-8,26]$. However, the functional role of TcES in regulating the activation and inflammatory response of macrophages remains unknown. In this study, we evaluated the effect of TcES on the polarization towards an M2 profile, inflammatory immune response, and transcriptomic profile of macrophages in vitro. 
$4 \mathrm{~h}$ post-stimulus
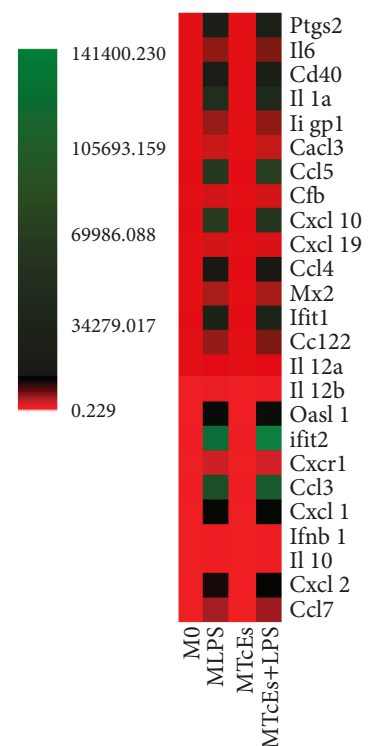
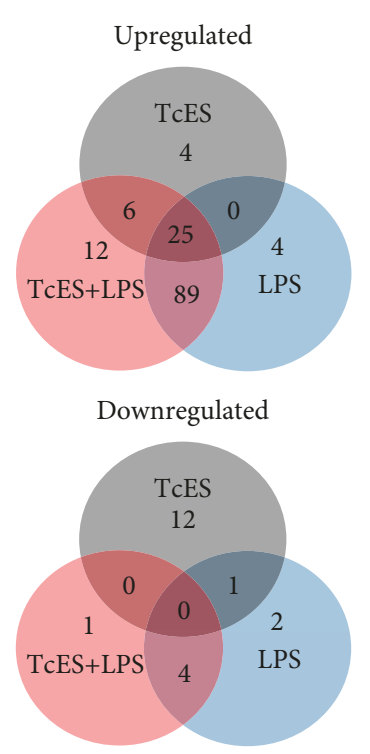

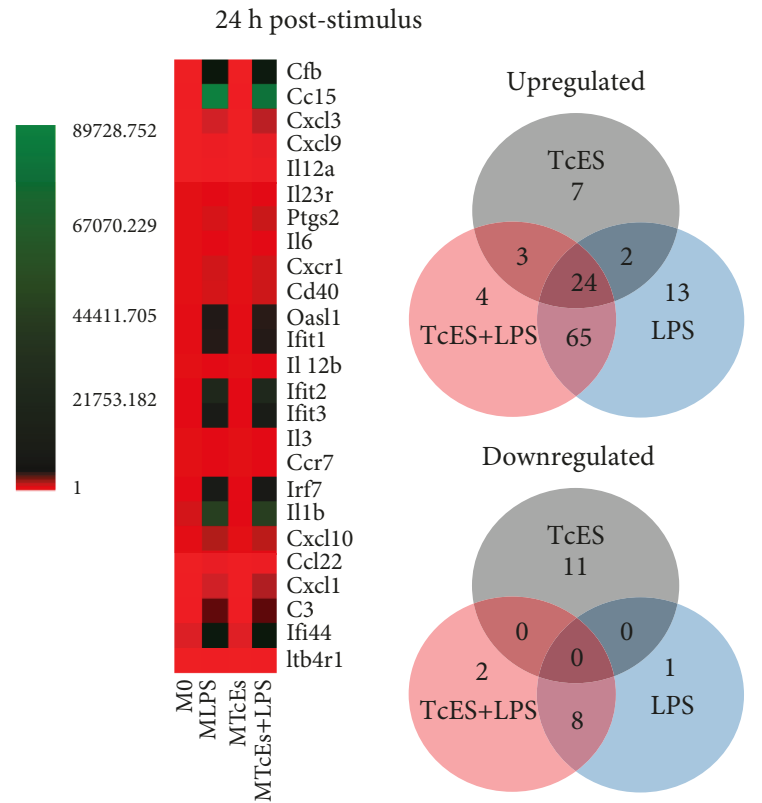

FIGURE 3: Top 25 upregulated mRNAs in stimulated BMDM. Heat maps show mRNA levels among $\mathrm{M}_{\mathrm{TcES}}, \mathrm{M}_{\mathrm{TcES}+\mathrm{LPS}}$, and $\mathrm{M}_{\mathrm{LPS}}$ at 4 and $24 \mathrm{~h}$ poststimulus. Each row represents mRNA levels, and each column represents a specific sample. The color scale illustrates the relative levels of mRNA: green, increased production; red, decreased production; and black, mean value. Venn diagrams show the unique and overlapping mRNA transcripts among the samples.

We first measure the production of the cytokines IL-6, IL-10, IL-12, and TNF $\alpha$ in BMDM-stimulated with TcES alone or in combination with LPS and observed that TcES increased the levels of the regulatory cytokine IL-10 and reduced the release of the inflammatory cytokines IL-6, IL-12, and TNF $\alpha$ in supernatants from LPS-stimulated BMDM. TcES alone did not increase the production of inflammatory cytokines but induced the release of IL-10 in BMDM. The levels of both mRNAs of Ill0 and Tnf measured by RT-qPCR showed similar trends when compared to the levels of cytokines obtained by ELISA assay, suggesting a consistent role for TcES in regulating cytokine production by inhibition of their transcripts.

Here, we evaluated the production of NOS2 and ARG1 in BMDM stimulated with TCES alone or in combination with IL-4 or LPS. M1 macrophages normally produce NOS2, which metabolizes L-arginine to nitric oxide (NO), while M2 macrophages produce ARG1, which metabolizes Larginine to produce prolines and polyamines $[2,27]$. We found that whereas BMDM stimulated with IL-4 or LPS alone showed increased levels of ARG1 and NOS2, respectively, TcES did not alter the production of both NOS2 and ARG1, after $24 \mathrm{~h}$ poststimulation. Our data are in agreement with previous studies using Fasciola hepatica tegumental antigens, which also failed to directly induce the production of molecules associated with M2 macrophages in vitro but not in vivo [28]. The production of M2 canonical molecules such as ARG1 has been reported to be IL-4-dependent, which is produced by $\mathrm{T}_{\mathrm{H}} 2 \mathrm{~T}$ cells, natural killer $\mathrm{T}$ cells, and basophils but not macrophages [29-31]. Therefore, helminth antigen stimulation alone is not enough to induce functional polarization of BMDM towards M2; however, they influence the inflammatory properties of these cells. Therefore, TcES do not induce the production of M2-associated molecules but counteract inflammatory response in macrophages in vitro.

Recent studies indicate a regulatory role for helminth antigens obtained from Trichinella spiralis, Spirometra erinaceieuropaei, Schistosoma mansoni, and Hymenolepis diminuta in reducing cytokine production and subsequent inflammation [32-41]. However, the analysis of a small number of inflammatory products and/or conventional M1 and M2 markers poorly describes the effect of these antigens in the proinflammatory profile of macrophages. Therefore, using array approaches (nCounter Inflammation Panel, NanoString mRNAs), we determined the levels of multiple mRNAs involved in macrophage inflammatory response. As expected, $\mathrm{M}_{\mathrm{TcES}}$ displayed a lower number of upregulated inflammatory mRNAs, when compared to $\mathrm{M}_{\mathrm{LPS}}$ at 4 (42 vs. 120 mRNAs) and $24 \mathrm{~h}$ poststimulus (36 vs. 104 mRNAs). $\mathrm{M}_{\text {TcES }}$ induced mRNAs associated with phagocytosis, M2 macrophage, and anti-inflammatory response. For instance, $\mathrm{M}_{\mathrm{TcES}}$ showed increased levels of Pkca mRNA necessary for the biogenesis of phagolysosomes [42]. In addition, $\mathrm{M}_{\mathrm{TCES}}$ increased levels of Irf3, C1s, and Ptgs mRNAs which have been previously associated with anti-inflammatory microenvironments and identified in M2 macrophages [43-53]. Although our results suggest that TcES induce mRNAs associated with M2 macrophages, the stimulus with these helminth-derived molecules is not enough to induce a full expression of all M2 markers in macrophages as observed in previously reported studies [28].

In contrast, $\mathrm{M}_{\mathrm{TCES}+\mathrm{LPS}}$ and $\mathrm{M}_{\mathrm{LPS}}$ shared more than 60 proinflammatory mRNAs at both 4 and $24 \mathrm{~h}$ 


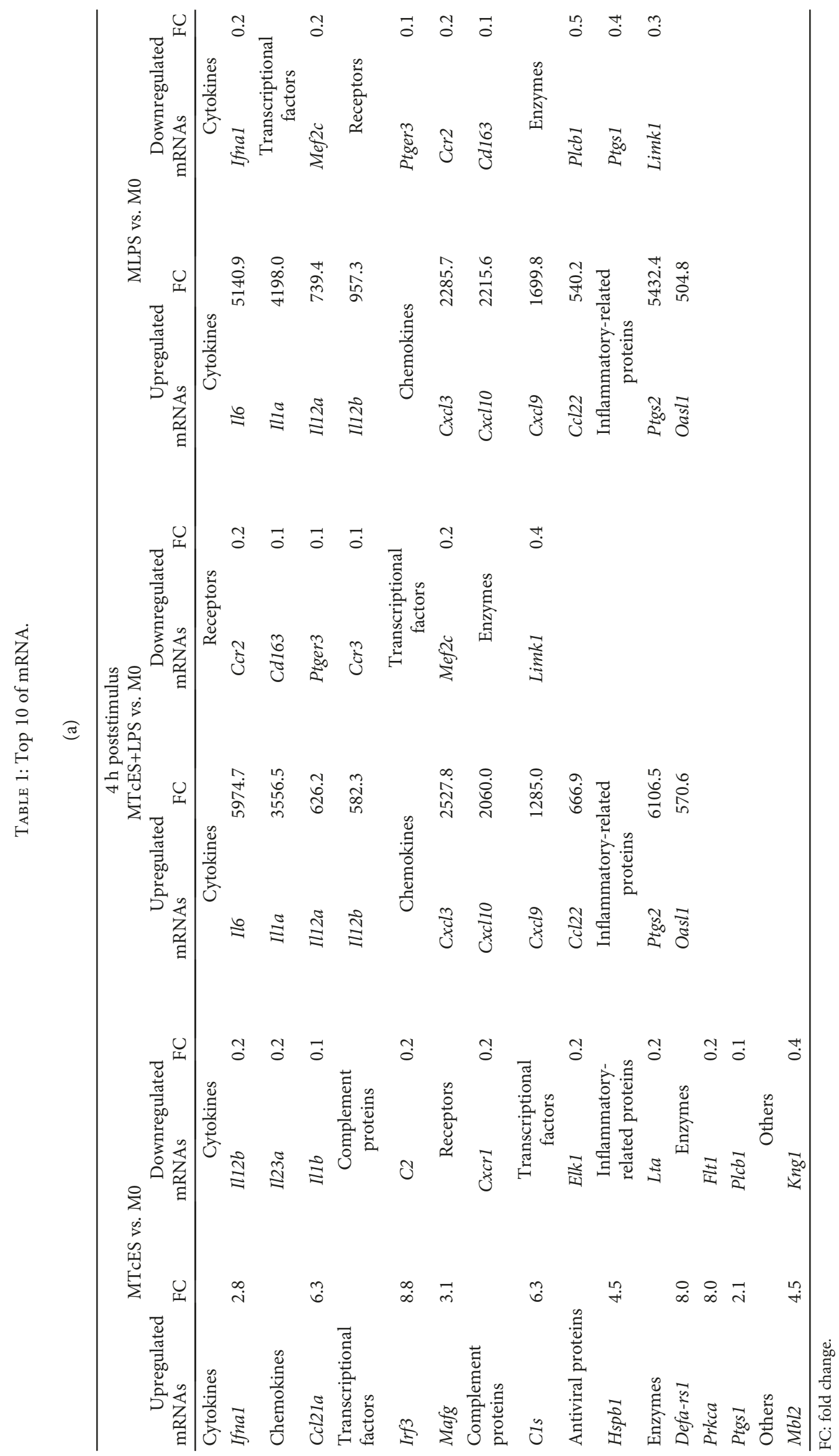




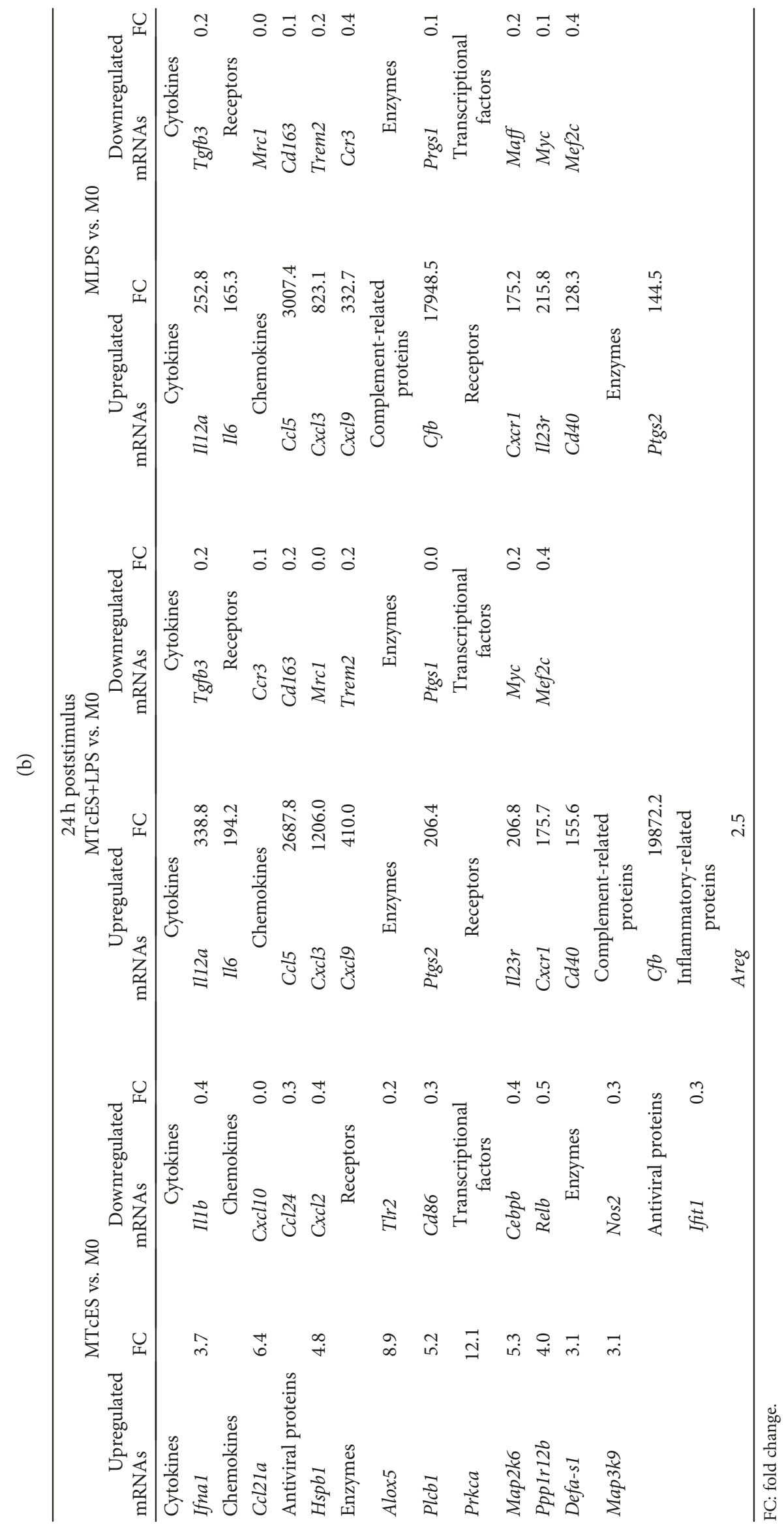



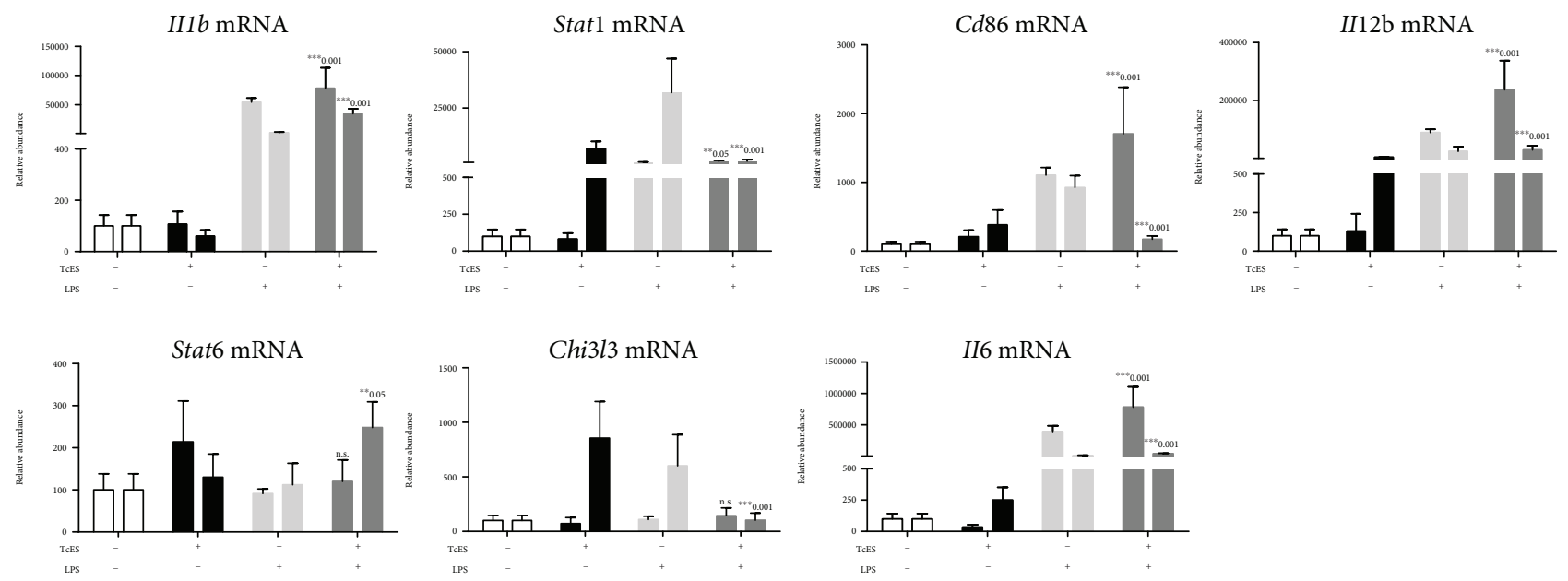

FIGURE 4: Validation and identification of mRNAs in stimulated BMDM. Macrophages were cultured in the presence of TcES $(25 \mu \mathrm{g} / \mathrm{mL})$, LPS $(1 \mu \mathrm{g} / \mathrm{mL}$ ), or both TcES+LPS for 4 (left bar) and $24 \mathrm{~h}$ (right bar) poststimulation. Relative levels of selected mRNA were determined by TaqMan mRNA assays after normalization with $18 \mathrm{~S}$ RNA. The levels of mRNA are represented as fold change relative to the PBStreated group $(\mathrm{FC} \pm \mathrm{SD})$. Data shown are representative of two independent experiments $(n=6,3$ replicates condition). Significance was calculated using $t$-test. ${ }^{*} p<0.01,{ }^{* *} p<0.05$, and ${ }^{* * *} p<0.001$.
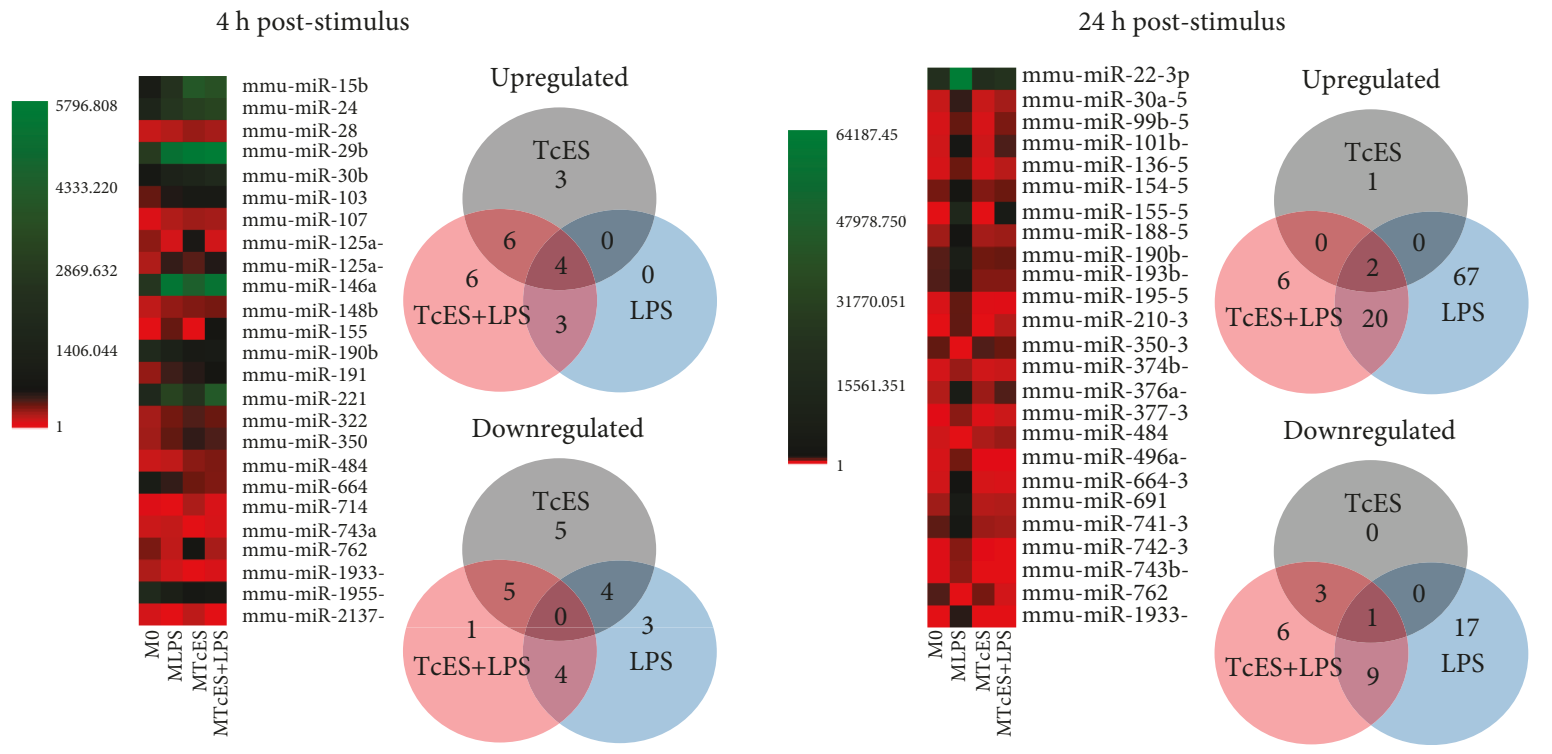

FIGURE 5: Differentially and commonly produced microRNAs in stimulated BMDM. The heat map shows the top 25 of microRNAs produced among $\mathrm{M}_{\mathrm{LPS}}, \mathrm{M}_{\mathrm{TCES}}$, and $\mathrm{M}_{\mathrm{TcES}+\mathrm{LPS}} 4 \mathrm{~h}$ and $24 \mathrm{~h}$ poststimulus. Each row represents a microRNA, and each column represents a specific sample. The color scale illustrates the relative level of microRNAs: green, increased production; red, decreased production; and black, mean value. Venn diagram showing the unique and overlapping microRNAs. A total of 22 and 96 modified microRNAs were found at 4 and $24 \mathrm{~h}$ poststimulation.

poststimulation. Interestingly, we observed reduced levels of different inflammatory mRNAs, e.g., Nox1, Ccl21a, Ccr4, and Cxcr2, in $\mathrm{M}_{\mathrm{TcES}+\mathrm{LPS}}$ with respect to $\mathrm{M}_{\mathrm{LPS}}$ at $24 \mathrm{~h}$ poststimulus. Noteworthily, although the levels of Il6, Il12a, and Il12b mRNAs were similar between $\mathrm{M}_{\mathrm{TcES}}$ +LPS and $\mathrm{M}_{\mathrm{LPS}}$, reduced levels of these cytokines were detected in supernatants from $\mathrm{M}_{\mathrm{TcES}+\mathrm{LPS}}$ with respect to $\mathrm{M}_{\text {LPS. }}$ A similar phenomenon has been reported for Acanthocheilonema viteae antigens, in decreasing $\mathrm{TNF} \alpha$ production in macrophages without altering Tnf transcripts [35], suggesting the participation of other posttranscriptional mechanisms. Additionally, $\mathrm{M}_{\mathrm{TcES}}$ and $\mathrm{M}_{\mathrm{TcES}+\mathrm{LPS}}$ shared levels of the mRNAs for Irf3, Defa1, C1s1, and Ifnal at $4 \mathrm{~h}$, and Hspb1, Maff, and Map2k6 at $24 \mathrm{~h}$ post stimulus. While levels of Irf3 and C1s1 mRNAs suggest an M2-like profile, levels of Ifna1 mRNA suggest that TcES could be recognized through TLR3, TLR7/8, or TLR9 $[54,55]$. To note, Defa 1 mRNA codifies protein HNP1 (human neutrophil- $\alpha$ defensin), which inhibits macrophage-driven inflammation through targeting proinflammatory cytokines and NO [56, 57]. Lastly, we noted that Tlr2 mRNA was upregulated in $\mathrm{M}_{\mathrm{TcES}+\mathrm{LPS}}$ 


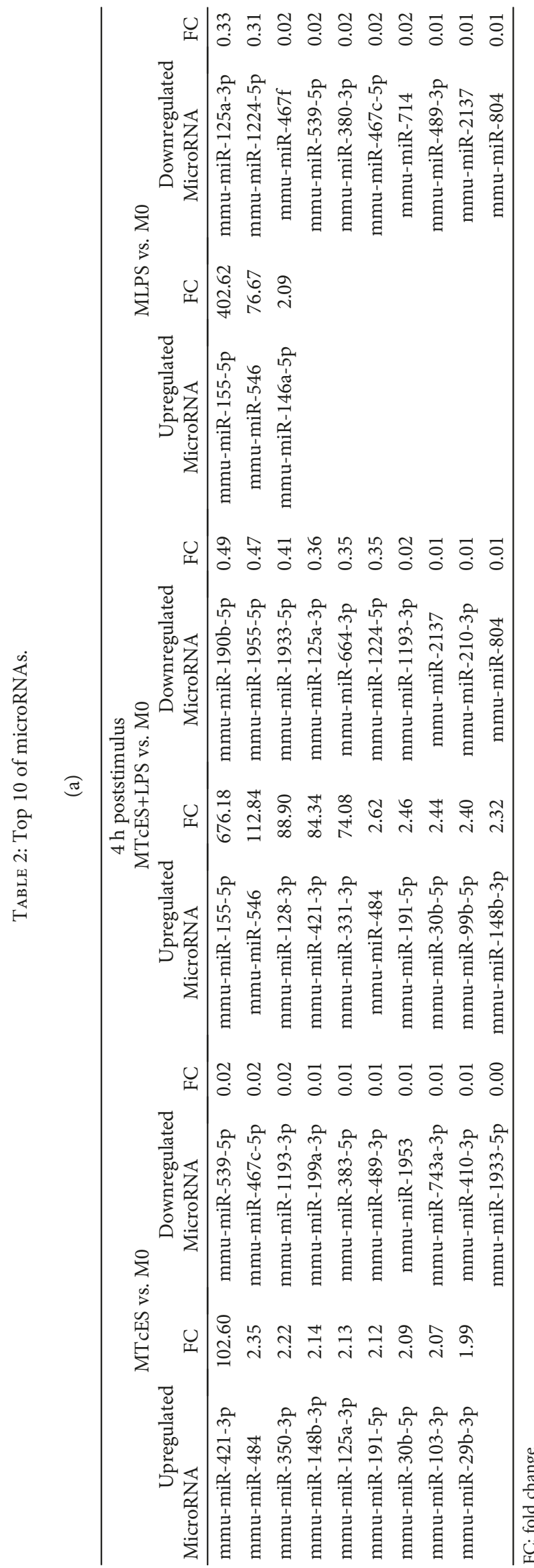

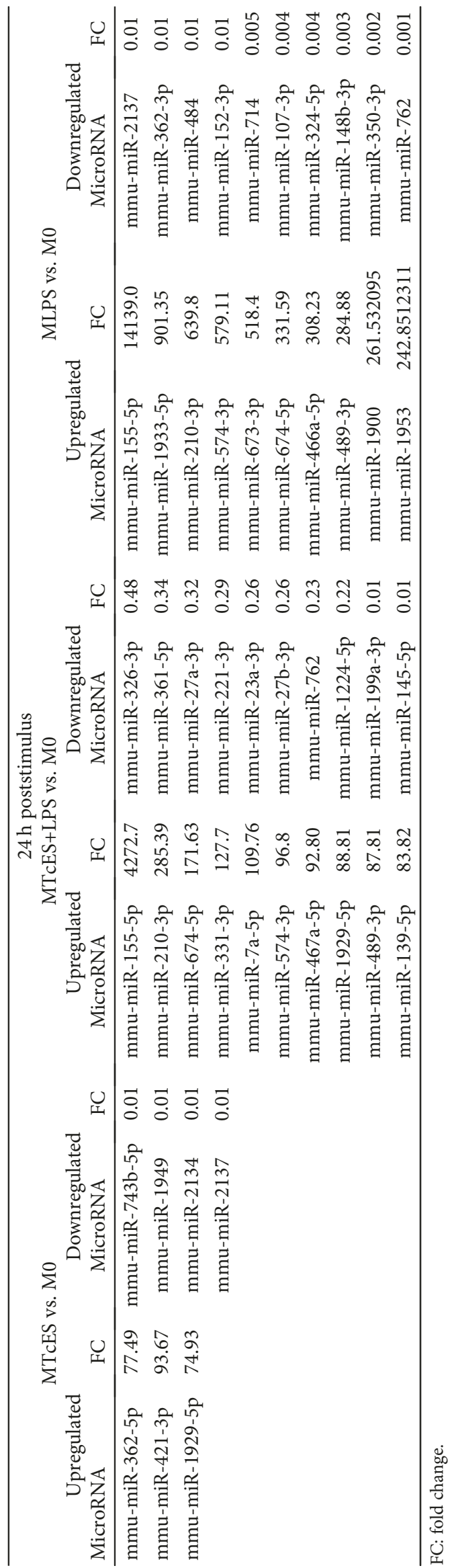


$\mathrm{M}_{\text {TcES }}$ VS. $\mathrm{M}_{0}$

Anatomical structure development

Cell differentiation

Embryo development

Cellular protein modification process

Cellular nitrogen compound metabolic process

Biosynthetic process

Chromosome organization

Anatomical structure formation involved in morphogenesis

Homeostatic process

Catabolic process

Cytoskeleton organization

cell morphogenesis

Cell cycle

Cell division

Cell death

Growth

Cell motility

Response to stress

Cellular component assembly

Developmental maturation

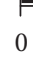

$$
\mathrm{M}_{\mathrm{TcES}} \text { VS. } \mathrm{M}_{0}
$$

Glioma

Chronic myeloid leukemia

TGF-beta signaling pathway

Insulin signaling pathway

Prostate cancer

HIF-1 signaling pathway

Arrhythmogenic right ventricular cardiomyopathy (ARVC)

ECM-receptor interaction

Inositol phosphate metabolism

Valine, leucine and isoleucine degradation

Colorectal cancer

Hepatitis B

Thyroid hormone signaling pathway

HTLV-I infection

Viral carcinogenesis

MAPK signaling pathway

Acute myeloid leukemia

AMPK signaling pathway

Glyoxylate and dicarboxylate metabolism

Pancreatic cancer

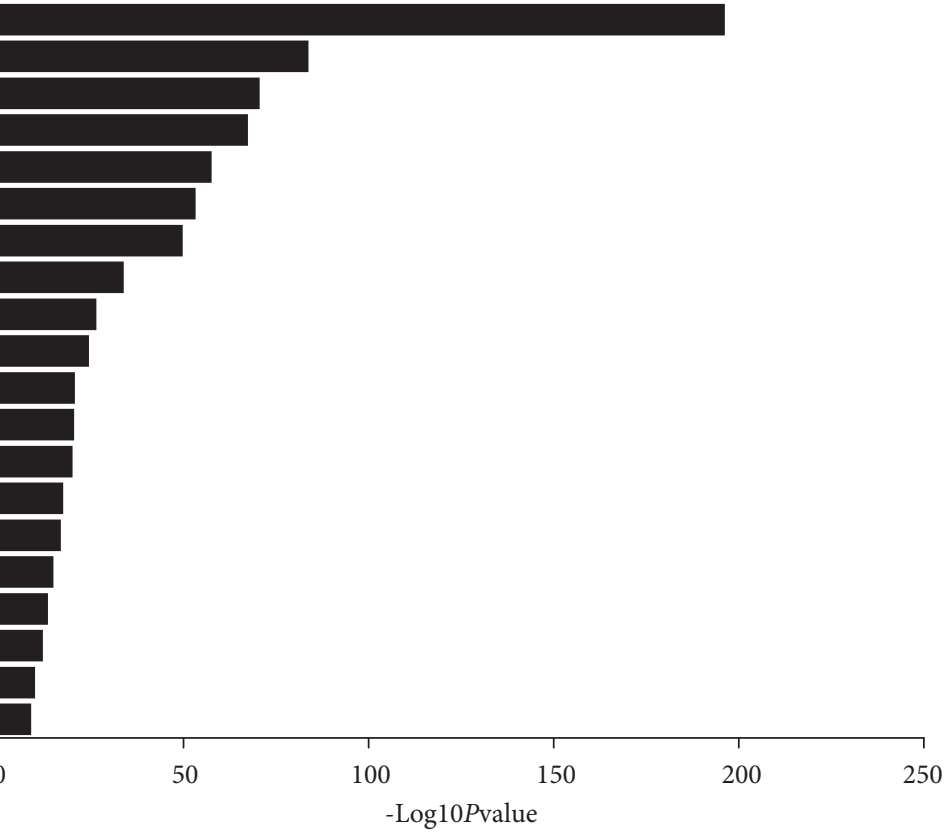

50

100

-Log10Pvalue

Biological process

KEGG pathways

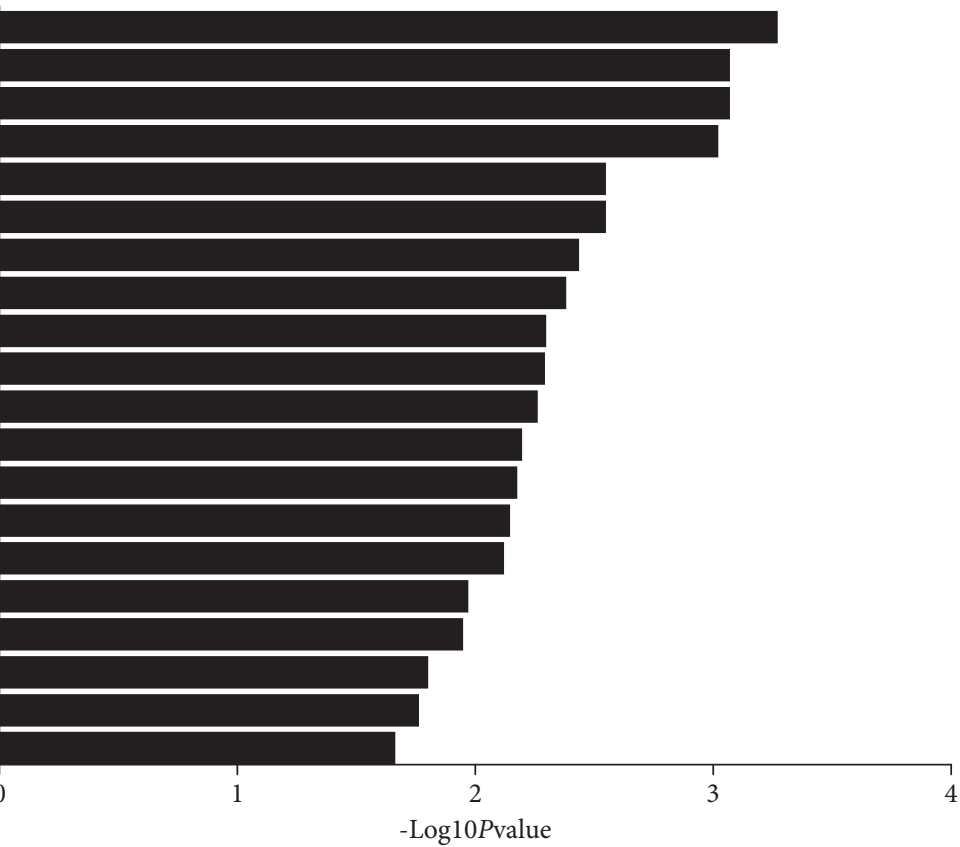

(a)

FIGURE 6: Continued. 
$\mathrm{M}_{\mathrm{TcES}+\mathrm{LPS}} \mathrm{VS} . \mathrm{M}_{0}$

Anatomical structure development

Cell differentiation

Embryo development

Cellular protein modification process Cellular nitrogen compound metabolic process

Biosynthetic process

Chromosome organization

Anatomical structure formation involved in morphogenesis

Homeostatic process

Catabolic process

Cytoskeleton organization

cell morphogenesis

Cell cycle

Cell division

Cell death

Growth

Cell motility

Response to stress

Cellular component assembly

Developmental maturation

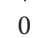

$\mathrm{M}_{\text {TcES+LPS }}$ VS. $\mathrm{M}_{0}$

Ubiquitin-mediated proteolysis

p53 signaling pathway

GnRH signaling pathway

$\mathrm{T}$ cell receptor signaling pathway Focal adhesion

Insulin signaling pathway

TNF signaling pathway

TGF-beta signaling pathway

Peroxisome

Prostate cancer

Inositol phosphate metabolism

HTLV-I infection

Pancreatic cancer

HIF-1 signaling pathway

Hepatitis B

Glioma

Phosphatidylinositol signaling system

Citrate cycle (TCA cycle)

Thyroid hormone synthesis

Progesterone-mediated oocyte maturation

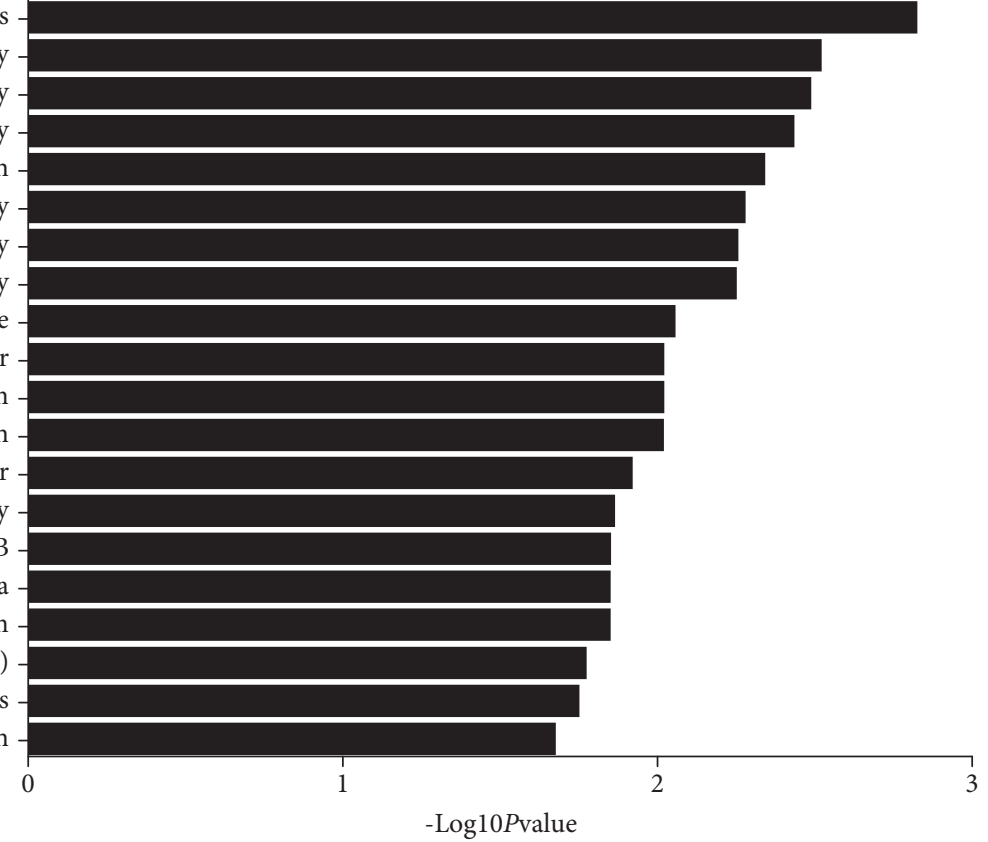

Figure 6: Continued. (b)

Biological process

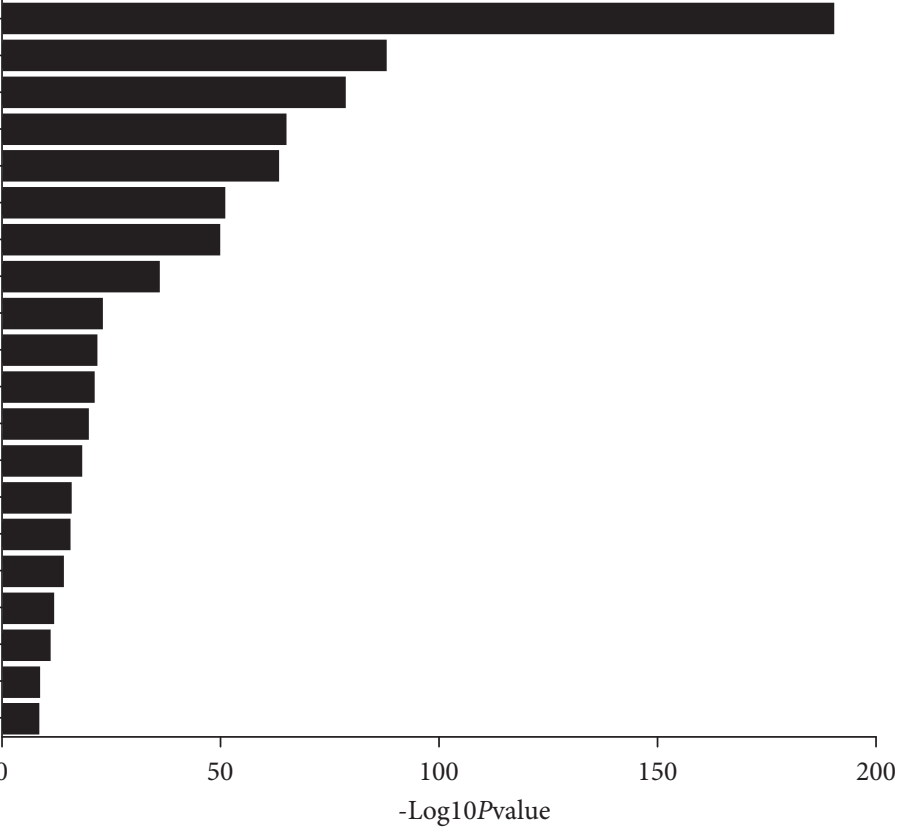

KEGG pathways 
$\mathrm{M}_{\text {LPS }}$ VS. $\mathrm{M}_{0}$

Chromosome organization

Biosynthetic process

Protein complex

Cell cycle

Cell division

Catabolic process

Anatomical structure formation involved in morphogenesis

Cell morphogenesis

$$
\text { Cell death }
$$

Chromosome

Homeostatic process

Cytoskeleton organization

Nuclear chromosome

Nucleic acid binding transcription factor activity

$$
\text { Nucleoplasm - }
$$

Cytoplasmic membrane-bounded vesicle

Response to stress

Enzyme binding

Protein binding transcription factor activity

Immune system process

$$
0
$$

$$
\mathrm{M}_{\text {LPS }} \text { VS. } \mathrm{M}_{0}
$$

Prostate cancer

Steroid biosynthesis

FoxO signaling pathway

Thyroid hormone synthesis

Acute myeloid leukemia

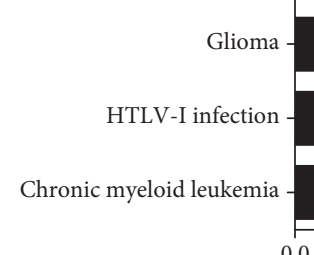

0.0
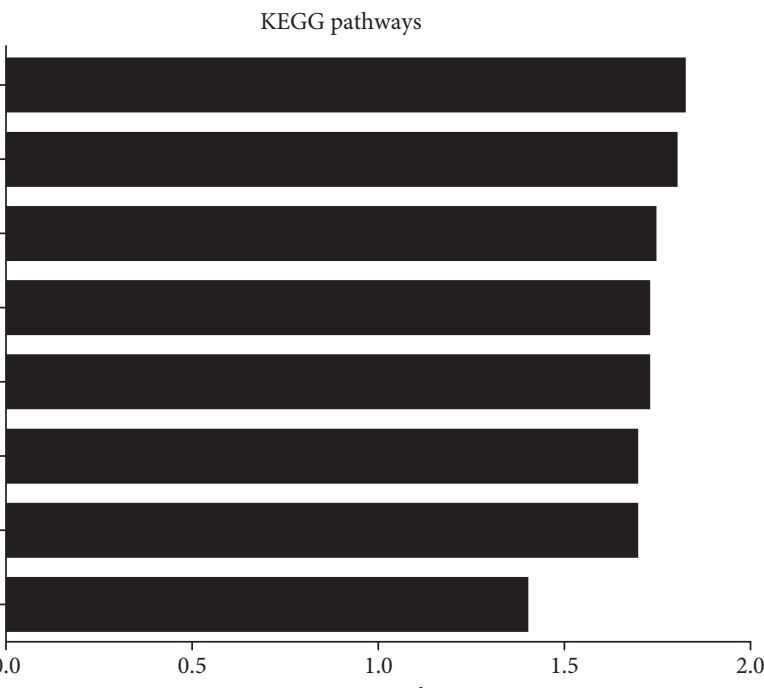

(c)

Anatomical structure development Cell differentiation Chromosome organization embryo development Cellular nitrogen compound metabolic process

Biosynthetic process Cellular protein modification process

Catabolic process

Cell morphogenesis Cell motility Growth Anatomical structure formation involved in morphogenesis In utero embryonic development

Cytoskeleton organization
Cell cycle Cell division

Cellular component assembly Chromosome segregation Negative regulation of translation Positive regulation of mitotic cell cycle

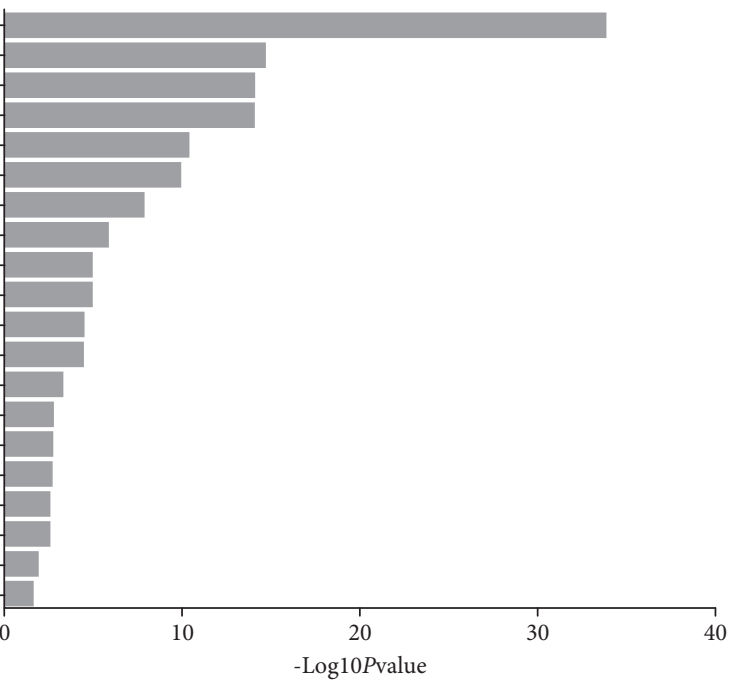

(d)

Figure 6: Continued. 

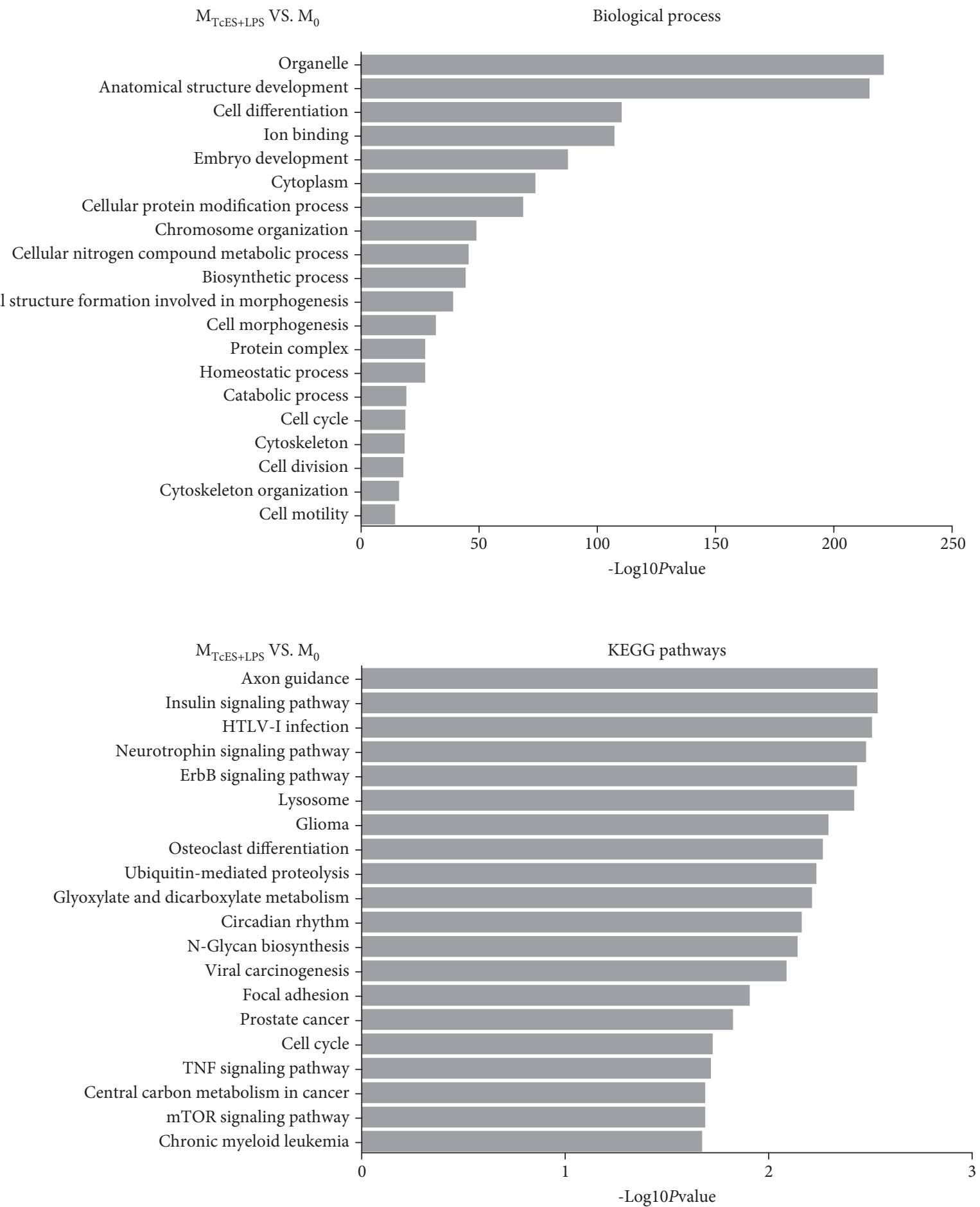

(e)

Figure 6: Continued. 


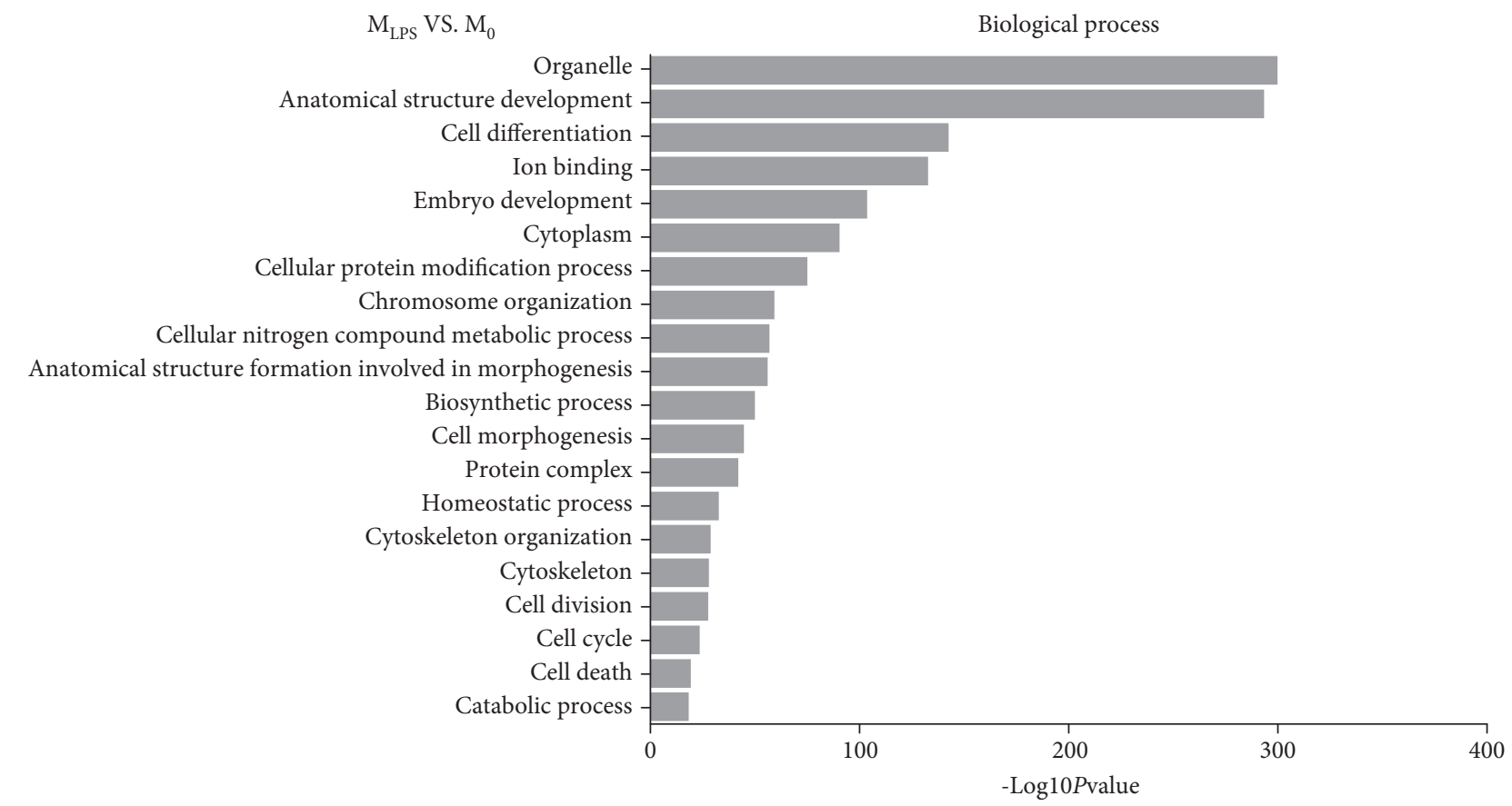

$\mathrm{M}_{\text {LPS }}$ VS. $\mathrm{M}_{0}$

KEGG pathways

Inositol phosphate metabolism

Pathways in cancer Insulin signaling pathway

Long-term depression Axon guidance

AMPK signaling pathway

T cell receptor signaling pathwa

TNF signaling pathway

HIF-1 signaling pathway Pancreatic cancer

$\mathrm{N}-$ Glycan biosynthesis

Citrate cycle (TCA cycle) Prostate cancer

Phosphatidylinositol signaling system Valine, leucine and isoleucine degradation

Propanoate metabolism

TGF-beta signaling pathway Regulation of actin cytoskeleton

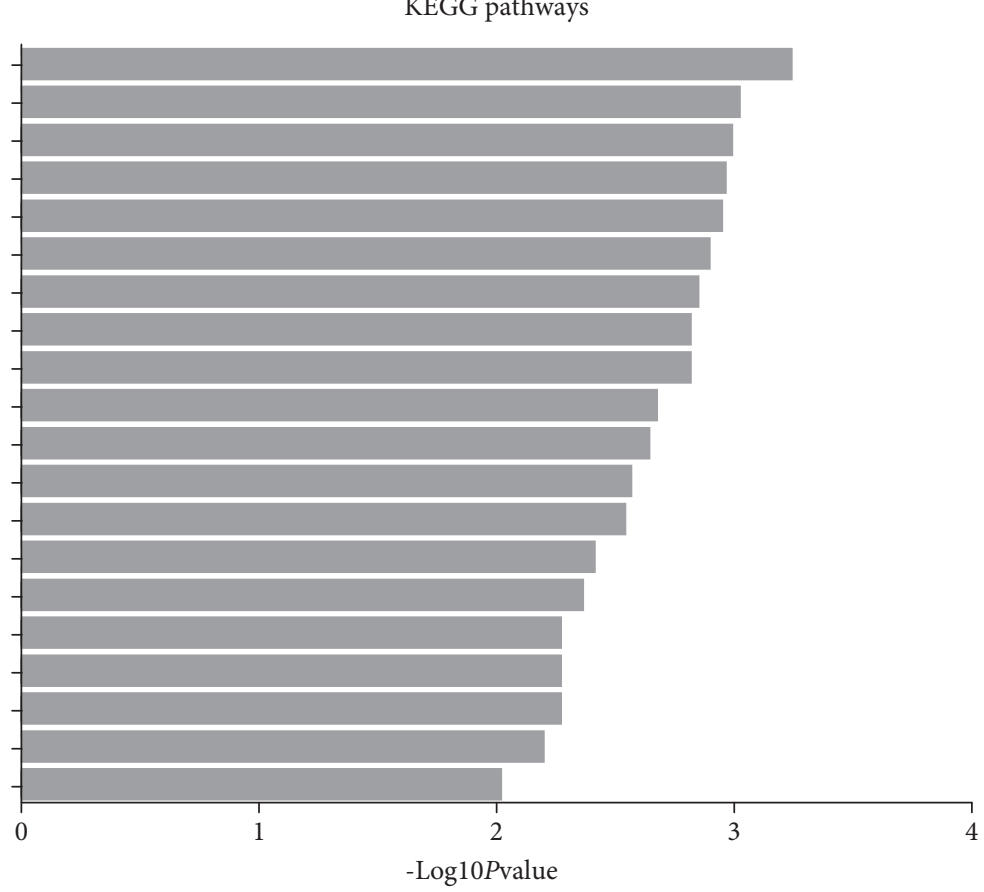

(f)

Figure 6: Top 20 of GO biological processes and KEGG pathways in $\mathrm{M}_{\mathrm{TcES}}, \mathrm{M}_{\mathrm{TcES}+\mathrm{LPS}}$, and $\mathrm{M}_{\mathrm{LPS}}$ at 4 (black) and $24 \mathrm{~h}$ poststimulus (gray). GO biological processes and KEGG pathways enriched by the upregulated differentially produced microRNAs between $\mathrm{M}_{\mathrm{TcES}} \mathrm{vs}_{\mathrm{S}} \mathrm{M}_{0}(\mathrm{a}$ and d), $\mathrm{M}_{\mathrm{TcES}+\mathrm{LPS}}$ vs. $\mathrm{M}_{0}$ (b and e), and $\mathrm{M}_{\mathrm{LPS}}$ vs. $\mathrm{M}_{0}$ (c and $\mathrm{f}$ ) at $4 \mathrm{~h}$ and $24 \mathrm{~h}$ poststimulus, respectively.

at $24 \mathrm{~h}$ post stimulus, which could be attributed to TcES's own recognition, as previously have been reported to recognize TcES [58]. These data suggest that posttranscriptional events may be involved in the regulatory mechanism triggered by TcES in regulating macrophage inflammatory responses.
microRNAs, small noncoding RNA molecules, have emerged as a key component of macrophage posttranscriptional regulation [59]. These molecules can silence the translation of mRNAs via base-pairing with complementary sequences within the RNA molecules. Hence, we further analyzed the microRNA profile in BMDM stimulated with 


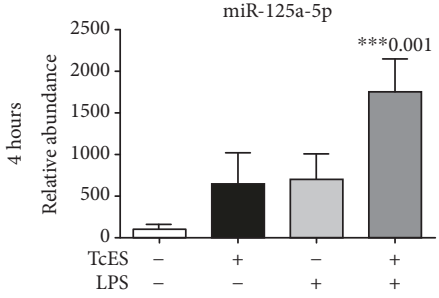

(a)

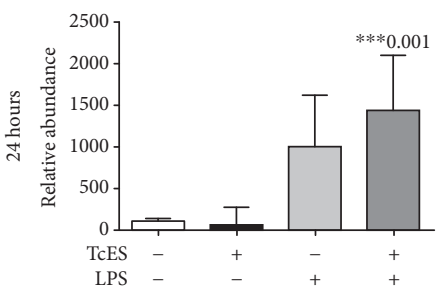

(b)

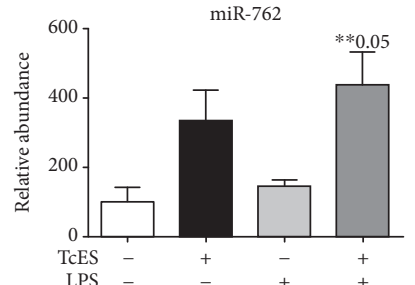

(c)

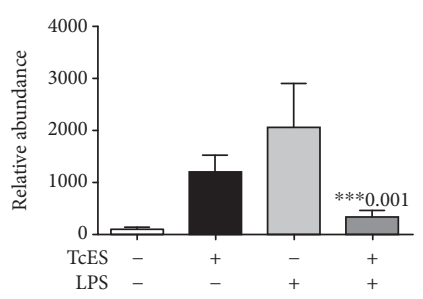

(d)

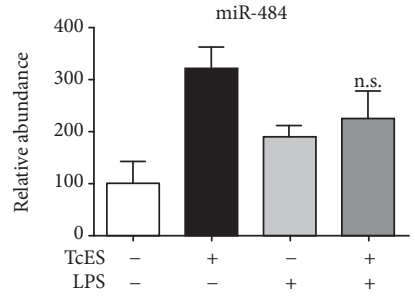

(e)

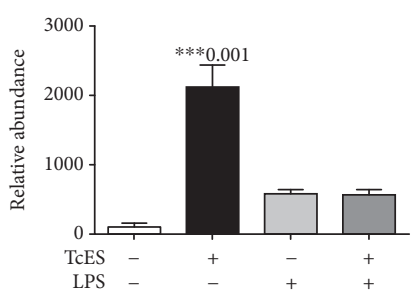

(f)

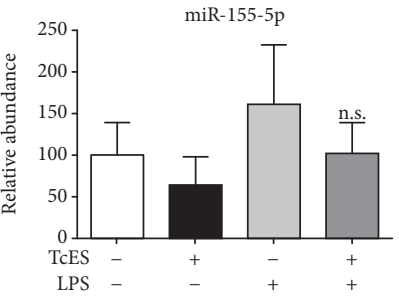

(g)

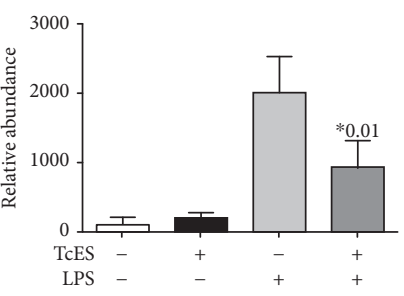

(h)

FIGURE 7: Validation and identification of microRNAs in stimulated BMDM. Macrophages were cultured in the presence of TcES (25 $\mu \mathrm{g} / \mathrm{mL})$, LPS $(1 \mu \mathrm{g} / \mathrm{mL})$, or a combination of TcES+LPS for 4 (left bar) and $24 \mathrm{~h}$ poststimulus (right bar). Relative levels of selected microRNAs were determined by TaqMan miRNA assays after normalization with 18S RNA. MicroRNA levels are represented as fold change relative to PBStreated BMDM $(\mathrm{FC} \pm \mathrm{SD})$. Data shown are representative of two independent experiments. Significance was calculated using $t$-test. * $p<0.01$, ${ }^{* *} p<0.05$, and ${ }^{* * *} p<0.001$.

TcES alone or in combination with LPS. Our analysis demonstrated that $\mathrm{M}_{\mathrm{TcES}+\mathrm{LPS}}$ shared regulatory microRNAs with $\mathrm{M}_{\mathrm{LPS}}$. For example, miR-146a-5p was upregulated in $\mathrm{M}_{\mathrm{TcES}+\mathrm{LPS}}$ and $\mathrm{M}_{\mathrm{LPS}}$ at $4 \mathrm{~h}$ and only in $\mathrm{M}_{\mathrm{TcES}+\mathrm{LPS}}$ at $24 \mathrm{~h}$ poststimulus. This microRNA has been reported to dampen proinflammatory responses in macrophages through the inhibition of TLRs, NF- $\kappa \mathrm{B}$, and STAT signaling pathways by targeting the mRNAs of Traf6, Irak1, Irak2, $N f \kappa b$, Stat1, and Ap1 [60-63]. This evidence is supported by our KEGG enrichment analysis, which indicates that overproduced microRNAs in $\mathrm{M}_{\mathrm{TcES}+\mathrm{LPS}}$ target mRNAs involved in NF- $\kappa \mathrm{B}, \mathrm{TNF}$, and MAPK signaling pathways. Of note, these data also confirm our hypothesis that TcES target proinflammatory pathways and support our previous findings indicating a role for TcES in blocking the IFN- $\gamma /$ STAT1 signaling pathway in macrophages in vitro [17].

$\mathrm{M}_{\text {TcES+LPS }}$ also overproduced microRNAs previously reported to target inflammatory mRNAs; for instance, let-7i and let-7e target Tlr4 mRNA, which causes a drop in the recognition of proinflammatory antigens [64-66]. Moreover, miR-24-3p production in macrophages has been reported to significantly decrease the production of IL- 6 and TNF $\alpha$ [67]. Furthermore, $\mathrm{M}_{\mathrm{TcES}+\mathrm{LPS}}$ and $\mathrm{M}_{\mathrm{TcES}}$ shared upregulated microRNAs previously reported to be elicited in macrophages exposed to E. multilocularis antigens (e.g., miR146a-5p) and S. japonicum (miR-365 and miR-24) [14, 68]. These data suggest the presence of conserved antigens among helminths that could trigger similar posttranscriptional mechanisms to modulate immune responses in the host.

Finally, we selected four upregulated microRNAs to validate their levels by RT-qPCR and confirm the high quality of our array. We observed increased levels of miR-125a-5p in $\mathrm{M}_{\mathrm{TcES}}$ and $\mathrm{M}_{\mathrm{LPS}}$, as early as $4 \mathrm{~h}$ poststimulus. The combined stimulus with TcES and LPS induced an additive effect in the levels of this microRNA. miR-125a-5p has been reported to increase after TLR2/4 signaling and has a key role in reducing the production of inflammatory cytokines (IL-6, IL-12, and TNF $\alpha$ ) by targeting NF- $\kappa \mathrm{B}$ and KFL4 signaling pathways [24, 69-71]. These data are associated with our previous studies suggesting that TcES is a ligand of TLR2 in phagocytic cells [58, 72]. In addition, miR-762 was selectively induced in $\mathrm{M}_{\mathrm{TcES}}$ and $\mathrm{M}_{\mathrm{TcES}+\mathrm{LPS}}$ at $4 \mathrm{~h}$ poststimulus. miR762 has been demonstrated to increase in ovarian and breast cancer and ocular tissue [73-75] where macrophages normally acquire an M2-like phenotype [76, 77]. Furthermore, by using bioinformatic tools, we found Il12b, Il6, Tnf, Nfkb, and Cd86 mRNAs as possible targets of miR-762 in $\mathrm{M}_{\mathrm{TcES}}$ and $\mathrm{M}_{\mathrm{TcES}+\mathrm{LPS}}$. The microRNA miR-484 was found to be upregulated in all the groups of stimulated BMDM at $4 \mathrm{~h}$; however, its levels were only sustained in $\mathrm{M}_{\mathrm{TcES}}$ at $24 \mathrm{~h}$ poststimulation. miR-484 has been previously identified in multiple types of cancers [78-82] and the cerebral cortex [83]; such microenvironments are known to promote an antiinflammatory phenotype in macrophages. Our bioinformatic analysis shows that Il1b, Nfkb, Stat5a, Irf1, Myd88, Stat1, and IL-12a mRNAs are possible targets for miR-484, which suggest a possible role for miR-484 in immune tolerance.

Lastly, we observed that miR-155-5p was upregulated in $\mathrm{M}_{\text {LPS }}$ at $4 \mathrm{~h}$ and $\mathrm{M}_{\mathrm{TcES}+\mathrm{LPS}}$ and $\mathrm{M}_{\mathrm{LPS}}$ at $24 \mathrm{~h}$ poststimulus. miR-155-5p is a well-defined microRNA induced by LPS in macrophages, which enhances the proinflammatory response by targeting the immunomodulatory mRNAs Ship1, Socs1, Il13r $\alpha$, and Clebp $\beta$ and increasing the half-life of $\operatorname{Tnf}[64,84-88]$. However, antigens of another helminth, Angiostrongylus cantonensis, also upregulated miR-155-5p [89]. Therefore, it would be of interest to further study the role of miR-155-5p during exposure to helminth antigens.

In summary, our study demonstrates a role for TcES in regulating the production of key inflammatory cytokines, possibly by inducing microRNAs that target inflammatory 
transcripts and promoting the release of IL-10 in macrophages. This phenomenon shapes the transcriptomic profile of macrophages and consequently the outcome of the immune response. Although we found clear associations between TcES-induced microRNAs and mRNAs involved in multiple inflammatory pathways as their targets, our study has the limitation that we did not prove a direct interaction between microRNAs and mRNAs. Therefore, future studies in our laboratory will focus on elucidating the functional roles and significance of the different microRNAs described here. These findings increase our understanding of how released molecules from helminths regulate inflammation and may offer new approaches for the treatment of autoimmune and inflammatory diseases.

\section{Abbreviations}

M2: Alternatively activated-like macrophages

Arg1: $\quad$ Arginase 1

EAE: Autoimmune encephalomyelitis

BMDM: Bone marrow-derived macrophages

M1: Classical activation in macrophages

DAMPs: Danger-associated molecular patterns

DMEM: Dulbecco's modified Eagle's media

FBS: $\quad$ Fetal bovine serum

FC: $\quad$ Fold change (FC)

Fizz1: Found in inflammatory zone

GO: $\quad$ Gene ontology

HNP1: Human neutrophil- $\alpha$ defensin

IL-4R $\alpha: \quad$ IL-4 receptor $\alpha$

IGFBP5: Insulin-like growth factor binding protein 5

IL-10: Interleukin-10

IL-12: Interleukin-12

IL-4: $\quad$ Interleukin-4

IL-6: Interleukin-6

KEGG: Kyoto Encyclopedia of Genes and Genomes

LPS: Lipopolysaccharide

M-CSF: Macrophage colony-stimulating factor

MHC: Major histocompatibility complex

MR: $\quad$ Mannose receptor

mRNAs: Messenger RNAs

NO: $\quad$ Nitric oxide

Nos2: Nitric oxide synthase

PAMPs: Pathogen-associated molecular patterns

PD-L2: Programmed death ligand 2

TcES: Taenia crassiceps-excreted/secreted antigens

TLR: Toll-like receptor

TNF $\alpha$ : Tumor necrosis factor $\alpha$

UTR: Untranslated region.

\section{Data Availability}

The array data used to support the findings of this study have been deposited in the GEO (Gene Expression Omnibus) database of the NCBI with the accession numbers GSE125170 for RNAm and GSE125171 for microRNA as part of the SuperSerie GSE125172 which are public once this article is published.

\section{Conflicts of Interest}

The authors have no financial or other conflicts to declare.

\section{Acknowledgments}

This work was supported by grants IN220316 from DGAPAPAPIIT-UNAM and A1-S-37879 from Consejo Nacional de Ciencia y Tecnología (CONACYT) to LIT and by the National Institute of Allergy and Infectious Diseases, grant R21-AI120013, to SP-S. Diana Martínez-Saucedo is a PhD student from Programa de Doctorado en Ciencias Biomédicas, Universidad Nacional Autónoma de México (UNAM), and was supported by CONACYT 289850. BEC and J.D.R.R. received support from CONACYT.

\section{Supplementary Materials}

Table S1: sequence of primers used in RT-qPCRs to amplify the specified mRNAs (A) or microRNAs (B). Table S2: mRNAs commonly upregulated ( $\geq 2$-fold change) in BMDM with TcES (MTcES), LPS (MLPS), or TcES+LPS (MTcES + LPS) at both 4 (left) or $24 \mathrm{~h}$ (right) poststimulus. Table S3: transcripts up (A) or downregulated (B) at 4 or $24 \mathrm{~h}$ poststimulus with LPS (MLPS). Table S4: transcripts up (A) or downregulated (B) at 4 or $24 \mathrm{~h}$ poststimulus with TcES (MTcES). Table S5: transcripts up- (A) or downregulated (B) at 4 or $24 \mathrm{~h}$ poststimulus with TcES+LPS (MTcES +LPS). Table S6: mRNAs expressed differently ( $\geq 2$ FC) between BMDM stimulated with TcES+LPS (MTcES+LPS) and with LPS (MLPS) at 4 (left) or $24 \mathrm{~h}$ (right). Table S7: microRNAs up- (A) or downregulated (B) at 4 or $24 \mathrm{~h}$ poststimulus with LPS (MLPS). Table S8: microRNAs up- (A) or downregulated (B) at 4 or $24 \mathrm{~h}$ poststimulus with TcES (MTcES). Table S9: microRNAs up- (A) or downregulated (B) at 4 (left) or $24 \mathrm{~h}$ (right) poststimulus with TcES+LPS (MTcES+LPS). Table S10: microRNAs upregulated ( $\geq 2$-fold change) in BMDM with TcES (MTcES), LPS (MLPS), and TcES+LPS (MTcES+LPS) at both 4 (left) or $24 \mathrm{~h}$ (right) poststimulus. Table S11: microRNAs expressed differently $(\geq 2$ FC) between BMDM with TcES+LPS (MTcES+LPS) and with LPS (MLPS) at 4 (left) or $24 \mathrm{~h}$ (right) poststimulus. Table S12: KEGG pathway analysis. Significantly enriched KEGG pathways $(p<0.05)$ are presented for BMDM with TcES (MTcES), TcES+LPS (MTcES+LPS), or LPS (MLPS) at 4 (left) or $24 \mathrm{~h}$ (right) poststimulus. Table S13: GO biological process annotations of the target genes of differentially expressed microRNAs. According to $p$ value, GO terms of biological process are shown in BMDM with TcES (MTcES), TcES+LPS (MTcES+LPS), or LPS (MLPS) at 4 (left) or $24 \mathrm{~h}$ (right) poststimulus. (Supplementary Materials)

\section{References}

[1] K. A. Jablonski, S. A. Amici, L. M. Webb et al., "Novel markers to delineate murine M1 and M2 macrophages," PLoS One, vol. 10, no. 12, article e0145342, 2015.

[2] S. Gordon and F. O. Martinez, "Alternative activation of macrophages: mechanism and functions," Immunity, vol. 32, no. 5 , pp. 593-604, 2010. 
[3] R. M. Maizels, "Parasitic helminth infections and the control of human allergic and autoimmune disorders," Clinical Microbiology and Infection, vol. 22, no. 6, pp. 481-486, 2016.

[4] A. N. Peón, Y. Ledesma-Soto, J. E. Olguín, M. Bautista-Donis, E. Sciutto, and L. I. Terrazas, "Helminth products potently modulate experimental autoimmune encephalomyelitis by downregulating neuroinflammation and promoting a suppressive microenvironment," Mediators of Inflammation, vol. 2017, Article ID 8494572, 16 pages, 2017.

[5] A. Espinoza-Jiménez, R. de Haro, and L. I. Terrazas, "Taenia crassiceps antigens control experimental type 1 diabetes by inducing alternatively activated macrophages," Mediators of Inflammation, vol. 2017, Article ID 8074329, 15 pages, 2017.

[6] A. Espinoza-Jiménez, I. Rivera-Montoya, R. CárdenasArreola, L. Morán, and L. I. Terrazas, "Taenia crassiceps infection attenuates multiple low-dose streptozotocin-induced diabetes," Journal of Biomedicine \& Biotechnology, vol. 2010, Article ID 850541, 11 pages, 2010.

[7] J. L. Reyes, A. F. Espinoza-Jiménez, M. I. González, L. Verdin, and L. I. Terrazas, "Taenia crassiceps infection abrogates experimental autoimmune encephalomyelitis," Cellular Immunology, vol. 267, no. 2, pp. 77-87, 2011.

[8] Y. Ledesma-Soto, B. E. Callejas, C. A. Terrazas et al., "Extraintestinal helminth infection limits pathology and proinflammatory cytokine expression during DSS-induced ulcerative colitis: a role for alternatively activated macrophages and prostaglandins," BioMed Research International, vol. 2015, Article ID 563425, 17 pages, 2015.

[9] A. N. Peón, A. Espinoza-Jiménez, and L. I. Terrazas, "Immunoregulation by Taenia crassiceps and its antigens," BioMed Research International, vol. 2013, Article ID 498583, 13 pages, 2013.

[10] J. L. Reyes, C. A. Terrazas, J. Alonso-Trujillo, N. van Rooijen, A. R. Satoskar, and L. I. Terrazas, "Early removal of alternatively activated macrophages leads to Taenia crassiceps cysticercosis clearance in vivo," International Journal for Parasitology, vol. 40, no. 6, pp. 731-742, 2010.

[11] L. I. Terrazas, D. Montero, C. A. Terrazas, J. L. Reyes, and M. Rodríguez-Sosa, "Role of the programmed Death-1 pathway in the suppressive activity of alternatively activated macrophages in experimental cysticercosis," International Journal for Parasitology, vol. 35, no. 13, pp. 1349-1358, 2005.

[12] F. O. Martinez and S. Gordon, "The M1 and M2 paradigm of macrophage activation: time for reassessment," F1000 Prime Reports, vol. 6, p. 13, 2014.

[13] P. Cai, G. N. Gobert, and D. P. McManus, "MicroRNAs in parasitic helminthiases: current status and future perspectives," Trends in Parasitology, vol. 32, no. 1, pp. 71-86, 2016.

[14] X. Guo and Y. Zheng, "MicroRNA expression profile in RAW264.7 macrophage cells exposed to Echinococcus multilocularis metacestodes," Parasitology, vol. 145, no. 3, pp. 416423, 2018.

[15] D. Rückerl, S. J. Jenkins, N. N. Laqtom et al., "Induction of IL-4R $\alpha$-dependent microRNAs identifies PI3K/Akt signaling as essential for IL-4-driven murine macrophage proliferation in vivo," Blood, vol. 120, no. 11, pp. 23072316, 2012

[16] D. P. Bartel, "MicroRNAs genomics, biogenesis, mechanism, and function," Cell, vol. 116, no. 2, pp. 281-297, 2004.
[17] M. Becerra-Díaz and L. I. Terrazas, "Taenia crassiceps infection and its excreted/secreted products inhibit STAT1 activation in response to IFN- $\gamma$," International Journal for Parasitology, vol. 44, no. 9, pp. 613-623, 2014.

[18] S. Shicheng and J. Liu, "Isolation of mouse bone marrowderived monocytes," 2015, PROTOCOL (Version 1) available at Protocol Exchange.

[19] C. Terrazas, J. de Dios Ruiz-Rosado, S. A. Amici et al., "Helminth-induced Ly6C $\mathrm{C}$ monocyte-derived alternatively activated macrophages suppress experimental autoimmune encephalomyelitis," Scientific Reports, vol. 7, no. 1, article 40814, 2017.

[20] NanoString Technologies I, nCounter: Expression Data Analysis Guide, 2012.

[21] NanoString Technologies I, nCounter ${ }^{\circledR}$ Data Analysis Guidelines for miRNA, 2010.

[22] I. S. Vlachos, K. Zagganas, M. D. Paraskevopoulou et al., "DIANA-miRPath v3.0: deciphering microRNA function with experimental support," Nucleic Acids Research, vol. 43, pp. W460-W466, 2015.

[23] X. Jin, X. Guo, D. Zhu, M. Ayaz, and Y. Zheng, "miRNA profiling in the mice in response to Echinococcus multilocularis infection," Acta Tropica, vol. 166, pp. 39-44, 2017.

[24] S. Banerjee, H. Cui, N. Xie et al., "miR-125a-5p regulates differential activation of macrophages and inflammation," The Journal of Biological Chemistry, vol. 288, no. 49, pp. 3542835436, 2013.

[25] Y. Li, R. Huang, L. Wang et al., "microRNA-762 promotes breast cancer cell proliferation and invasion by targeting IRF7 expression," Cell Proliferation, vol. 48, no. 6, pp. 643649, 2015.

[26] S. León-Cabrera, B. E. Callejas, Y. Ledesma-Soto et al., "Extraintestinal helminth infection reduces the development of colitis-associated tumorigenesis," International Journal of Biological Sciences, vol. 10, no. 9, pp. 948-956, 2014.

[27] M. Rath, I. Müller, P. Kropf, E. I. Closs, and M. Munder, "Metabolism via arginase or nitric oxide synthase: two competing arginine pathways in macrophages," Frontiers in Immunology, vol. 5, p. 532, 2014.

[28] P. N. Adams, A. Aldridge, K. V. Vukman, S. Donnelly, and S. M. O'Neill, "Fasciola hepatica tegumental antigens indirectly induce an M2 macrophage-like phenotype in vivo," Parasite Immunology, vol. 36, no. 10, pp. 531-539, 2014.

[29] S. Huber, R. Hoffmann, F. Muskens, and D. Voehringer, "Alternatively activated macrophages inhibit T cell proliferation by STAT6-dependent expression of PD-L2," Blood, vol. 116, no. 17, pp. 3311-3320, 2010.

[30] P. G. Fallon, H. E. Jolin, P. Smith et al., "IL-4 induces characteristic Th2 responses even in the combined absence of IL-5, IL-9, and IL-13," Immunity, vol. 17, no. 1, pp. 7-17, 2002.

[31] T. Yoshimoto, "The hunt for the source of primary interleukin-4: how we discovered that natural killer T cells and basophils determine $\mathrm{T}$ helper type 2 cell differentiation In Vivo," Frontiers in Immunology, vol. 9, p. 716, 2018.

[32] P. Dirgahayu, S. Fukumoto, K. Miura, and K. Hirai, "Excretory/secretory products from plerocercoids of Spirometra erinaceieuropaei suppress the TNF- $\alpha$ gene expression by reducing phosphorylation of ERK1/2 and p38 MAPK in macrophages," International Journal for Parasitology, vol. 32, no. 9, pp. 1155$1162,2002$. 
[33] L. Chen, K. V. N. Rao, Y.-X. He, and K. Ramaswamy, "Skinstage Schistosomula of Schistosoma mansoni produce an apoptosis-inducing factor that can cause apoptosis of T cells," The Journal of Biological Chemistry, vol. 277, no. 37, pp. 34329-34335, 2002.

[34] K. Ramaswamy, B. Salafsky, S. Potluri, Y. He, J. W. Li, and T. Shibuya, "Secretion of an anti-inflammatory, immunomodulatory factor by Schistosomulae of Schistosoma mansoni," Journal of Inflammation, vol. 46, no. 1, pp. 13-22, 1995.

[35] H. S. Goodridge, E. H. Wilson, W. Harnett, C. C. Campbell, M. M. Harnett, and F. Y. Liew, "Modulation of macrophage cytokine production by ES-62, a secreted product of the filarial nematode Acanthocheilonema viteae," Journal of Immunology, vol. 167, no. 2, pp. 940-945, 2001.

[36] X. Bai, X. Wu, X. Wang et al., "Regulation of cytokine expression in murine macrophages stimulated by excretory/secretory products from Trichinella spiralis in vitro," Molecular and Cellular Biochemistry, vol. 360, no. 1-2, pp. 79-88, 2012.

[37] A. Chauhan, Y. Sun, B. Pani et al., "Helminth induced suppression of macrophage activation is correlated with inhibition of calcium channel activity," PLoS One, vol. 9, no. 7, article e101023, 2014.

[38] S. Donnelly, C. M. Stack, S. M. O'Neill, A. A. Sayed, D. L. Williams, and J. P. Dalton, "Helminth 2-Cys peroxiredoxin drives Th2 responses through a mechanism involving alternatively activated macrophages," The FASEB Journal, vol. 22, no. 11, pp. 4022-4032, 2008.

[39] C. M. Hamilton, D. J. Dowling, C. E. Loscher, R. M. Morphew, P. M. Brophy, and S. M. O'Neill, "The fasciola hepatica tegumental antigen suppresses dendritic cell maturation and function," Infection and Immunity, vol. 77, no. 6, pp. 2488-2498, 2009.

[40] S. Almeida, P. Nejsum, and A. R. Williams, "Modulation of human macrophage activity by Ascaris antigens is dependent on macrophage polarization state," Immunobiology, vol. 223, no. 4-5, pp. 405-412, 2018.

[41] A. Zawistowska-Deniziak, K. Basałaj, B. Strojny, and D. Młocicki, "New data on human macrophages polarization by hymenolepis diminuta tapeworm - an in vitro study," Frontiers in Immunology, vol. 8, p. 148, 2017.

[42] J. D. Ng Yan Hing, M. Desjardins, and A. Descoteaux, "Proteomic analysis reveals a role for protein kinase C- $\alpha$ in phagosome maturation," Biochemical and Biophysical Research Communications, vol. 319, no. 3, pp. 810-816, 2004.

[43] F. O. Martinez, L. Helming, R. Milde et al., "Genetic programs expressed in resting and IL-4 alternatively activated mouse and human macrophages: Similarities and differences," Blood, vol. 121, no. 9, pp. e57-e69, 2013.

[44] M. Rehli, S. Sulzbacher, S. Pape et al., "Transcription factor Tfec contributes to the IL-4-inducible expression of a small group of genes in mouse macrophages including the granulocyte colony-stimulating factor receptor," The Journal of Immunology, vol. 174, no. 11, pp. 7111-7122, 2005.

[45] W. H. Busby Jr, T.-J. Nam, A. Moralez, C. Smith, M. Jennings, and D. R. Clemmons, "The complement component C1s is the protease that accounts for cleavage of insulin-like growth factor-binding protein-5 in fibroblast medium," The Journal of Biological Chemistry, vol. 275, no. 48, pp. 37638-37644, 2000.

[46] F. O. Martinez, S. Gordon, M. Locati, and A. Mantovani, "Transcriptional profiling of the human monocyte-tomacrophage differentiation and polarization: new molecules and patterns of gene expression," The Journal of Immunology, vol. 177, no. 10, pp. 7303-7311, 2006.

[47] A. J. Fleetwood, H. Dinh, A. D. Cook, P. J. Hertzog, and J. A. Hamilton, "GM-CSF- and M-CSF-dependent macrophage phenotypes display differential dependence on type I interferon signaling," Journal of Leukocyte Biology, vol. 86, no. 2, pp. 411-421, 2009.

[48] S. K. Biswas and A. Mantovani, "Macrophage plasticity and interaction with lymphocyte subsets: cancer as a paradigm," Nature Immunology, vol. 11, no. 10, pp. 889-896, 2010.

[49] C. Luo, M. Chen, A. Madden, and H. Xu, "Expression of complement components and regulators by different subtypes of bone marrow-derived macrophages," Inflammation, vol. 35, no. 4, pp. 1448-1461, 2012.

[50] A. T. Naito, T. Sumida, S. Nomura et al., "Complement C1q activates canonical Wnt signaling and promotes agingrelated phenotypes," Cell, vol. 149, no. 6, pp. 1298-1313, 2012.

[51] Y. Cai, B. H. D. Teo, J. G. Yeo, and J. Lu, "C1q protein binds to the apoptotic nucleolus and causes $\mathrm{C} 1$ protease degradation of nucleolar proteins," Journal of Biological Chemistry, vol. 290, no. 37, pp. 22570-22580, 2015.

[52] F. K. Kerr, G. O'Brien, N. S. Quinsey et al., "Elucidation of the substrate specificity of the $\mathrm{C} 1 \mathrm{~s}$ protease of the classical complement pathway," The Journal of Biological Chemistry, vol. 280, no. 47, pp. 39510-39514, 2005.

[53] H. Eriksson and M. H. Nissen, "Proteolysis of the heavy chain of major histocompatibility complex class I antigens by complement component C1s," Biochimica et Biophysica Acta (BBA) - Protein Structure and Molecular Enzymology, vol. 1037, no. 2, pp. 209-215, 1990.

[54] F. Bouchonnet, N. Boechat, M. Bonay, and A. J. Hance, "Alpha/beta interferon impairs the ability of human macrophages to control growth of mycobacterium bovis bcg," Infection and Immunity, vol. 70, no. 6, pp. 3020-3025, 2002.

[55] Y. Kumagai, O. Takeuchi, H. Kato et al., "Alveolar macrophages are the primary interferon- $\alpha$ producer in pulmonary infection with RNA viruses," Immunity, vol. 27, no. 2, pp. 240-252, 2007.

[56] M. Brook, G. H. Tomlinson, K. Miles et al., "Neutrophilderived alpha defensins control inflammation by inhibiting macrophage mRNA translation," Proceedings of the National Academy of Sciences of the United States of America, vol. 113, no. 16, pp. 4350-4355, 2016.

[57] J. Wehkamp, M. Schmid, and E. F. Stange, "Defensins and other antimicrobial peptides in inflammatory bowel disease," Current Opinion in Gastroenterology, vol. 23, no. 4, pp. 370378, 2007.

[58] J. L. Reyes, M. I. González, Y. Ledesma-Soto, A. R. Satoskar, and L. I. Terrazas, "TLR2 mediates immunity to experimental cysticercosis," International Journal of Biological Sciences, vol. 7, no. 9, pp. 1323-1333, 2011.

[59] J. B. Self-Fordham, A. R. Naqvi, J. R. Uttamani, V. Kulkarni, and S. Nares, "MicroRNA: dynamic regulators of macrophage polarization and plasticity," Frontiers in Immunology, vol. 8, p. 1062, 2017.

[60] C. G. R. Perry, G. J. F. Heigenhauser, A. Bonen, and L. L. Spriet, "High-intensity aerobic interval training increases fat and carbohydrate metabolic capacities in human skeletal muscle," Applied Physiology, Nutrition, and Metabolism, vol. 33, no. 6, pp. 1112-1123, 2008. 
[61] K. D. Taganov, M. P. Boldin, K. J. Chang, and D. Baltimore, "NF-kappaB-dependent induction of microRNA miR-146, an inhibitor targeted to signaling proteins of innate immune responses," Proceedings of the National Academy of Sciences of the United States of America, vol. 103, no. 33, pp. 1248112486, 2006.

[62] C. Perske, N. Lahat, S. S. Levin, H. Bitterman, B. Hemmerlein, and M. A. Rahat, "Loss of inducible nitric oxide synthase expression in the mouse renal cell carcinoma cell line RENCA is mediated by microRNA miR-146a," The American Journal of Pathology, vol. 177, no. 4, pp. 2046-2054, 2010.

[63] A. R. Naqvi, S. Zhong, H. Dang, J. B. Fordham, S. Nares, and A. Khan, "Expression profiling of LPS responsive miRNA in primary human macrophages," Journal of Microbial \& Biochemical Technology, vol. 8, no. 2, pp. 136-143, 2016.

[64] E. Tili, J.-J. Michaille, A. Cimino et al., "Modulation of miR155 and miR-125b levels following lipopolysaccharide/TNF$\alpha$ stimulation and their possible roles in regulating the response to endotoxin shock," The Journal of Immunology, vol. 179, no. 8, pp. 5082-5089, 2007.

[65] X.-M. Chen, P. L. Splinter, S. P. O'Hara, and N. F. LaRusso, "A cellular micro-RNA, let-7i, regulates toll-like receptor 4 expression and contributes to cholangiocyte immune responses against Cryptosporidium parvum infection," The Journal of Biological Chemistry, vol. 282, no. 39, pp. 2892928938, 2007.

[66] A. J. Murphy, P. M. Guyre, and P. A. Pioli, "Estradiol suppresses NF- $\kappa$ B activation through coordinated regulation of let-7a and miR-125b in primary human macrophages," The Journal of Immunology, vol. 184, no. 9, pp. 50295037, 2010.

[67] Z. Jingjing, Z. Nan, W. Wei et al., "MicroRNA-24 modulates Staphylococcus aureus-induced macrophage polarization by suppressing CHI3L1," Inflammation, vol. 40, no. 3, pp. 9951005, 2017.

[68] H. Han, J. Peng, Y. Hong et al., "MicroRNA expression profile in different tissues of BALB/c mice in the early phase of Schistosoma japonicum infection," Molecular and Biochemical Parasitology, vol. 188, no. 1, pp. 1-9, 2013.

[69] X. Zhao, Y. Tang, B. Qu et al., "MicroRNA-125a contributes to elevated inflammatory chemokine RANTES levels via targeting KLF13 in systemic lupus erythematosus," Arthritis \& Rheumatism, vol. 62, no. 11, pp. 3425-3435, 2010.

[70] T. Chen, Z. Huang, L. Wang et al., "MicroRNA-125a-5p partly regulates the inflammatory response, lipid uptake, and ORP9 expression in oxLDL-stimulated monocyte/macrophages," Cardiovascular Research, vol. 83, no. 1, pp. 131139, 2009.

[71] D. W. Melton, X. Lei, J. A. L. Gelfond, and P. K. Shireman, "Dynamic macrophage polarization-specific miRNA patterns reveal increased soluble VEGF receptor 1 by miR-125a-5p inhibition," Physiological Genomics, vol. 48, no. 5, pp. 345360, 2016.

[72] C. A. Terrazas, M. Alcántara-Hernández, L. Bonifaz, L. I. Terrazas, and A. R. Satoskar, "Helminth-excreted/secreted products are recognized by multiple receptors on DCs to block the TLR response and bias Th2 polarization in a cRAF dependent pathway," The FASEB Journal, vol. 27, no. 11, pp. 4547-4560, 2013.

[73] R. Hou, Z. Yang, S. Wang et al., "miR-762 can negatively regulate menin in ovarian cancer," OncoTargets and Therapy, vol. 10, pp. 2127-2137, 2017.
[74] M. Karali, I. Peluso, V. A. Gennarino et al., "miRNeye: a microRNA expression atlas of the mouse eye," BMC Genomics, vol. 11, no. 1, p. 715, 2010.

[75] Y. Shi, Y. Jia, W. Zhao, L. Zhou, X. Xie, and Z. Tong, "Histone deacetylase inhibitors alter the expression of molecular markers in breast cancer cells via microRNAs," International Journal of Molecular Medicine, vol. 42, no. 1, pp. 435-442, 2018.

[76] C. E. Lewis and J. W. Pollard, "Distinct role of macrophages in different tumor microenvironments," Cancer Research, vol. 66, no. 2, pp. 605-612, 2006.

[77] J. Mun, C. Tam, G. Chan, J. H. Kim, D. Evans, and S. Fleiszig, "MicroRNA-762 is upregulated in human corneal epithelial cells in response to tear fluid and Pseudomonas aeruginosa antigens and negatively regulates the expression of host defense genes encoding RNase7 and ST2," PLoS One, vol. 8, no. 2, article e57850, 2013.

[78] E. Pashaei, E. Pashaei, M. Ahmady, M. Ozen, and N. Aydin, "Meta-analysis of miRNA expression profiles for prostate cancer recurrence following radical prostatectomy," PLoS One, vol. 12, no. 6, article e0179543, 2017.

[79] V. Sansoni, S. Perego, G. Vernillo et al., "Effects of repeated sprints training on fracture risk-associated miRNA," Oncotarget, vol. 9, no. 26, pp. 18029-18040, 2018.

[80] E. Seclaman, D. Narita, A. Anghel et al., "MicroRNA expression in laser micro-dissected breast cancer tissue samples - a pilot study," Pathology \& Oncology Research, vol. 25, no. 1, pp. 233-239, 2019.

[81] Y. Hu, H. Xie, Y. Liu, W. Liu, M. Liu, and H. Tang, "miR-484 suppresses proliferation and epithelial-mesenchymal transition by targeting ZEB1 and SMAD2 in cervical cancer cells," Cancer Cell International, vol. 17, p. 36, 2017.

[82] S. Zearo, E. Kim, Y. Zhu et al., "MicroRNA-484 is more highly expressed in serum of early breast cancer patients compared to healthy volunteers," BMC Cancer, vol. 14, no. 1, p. 200, 2014.

[83] R. Yi, J. Feng, S. Yang et al., "miR-484/MAP2/c-Myc-positive regulatory loop in glioma promotes tumor-initiating properties through ERK1/2 signaling," Journal of Molecular Histology, vol. 49, no. 2, pp. 209-218, 2018.

[84] M. He, Z. Xu, T. Ding, D.-M. Kuang, and L. Zheng, "MicroRNA-155 regulates inflammatory cytokine production in tumor-associated macrophages via targeting C/EBP $\beta$," Cellular and Molecular Immunology, vol. 6, no. 5, pp. 343-352, 2009.

[85] B. Tang, B. Xiao, Z. Liu et al., "Identification of MyD88 as a novel target of miR-155, involved in negative regulation of Helicobacter pylori-induced inflammation," FEBS Letters, vol. 584, no. 8, pp. 1481-1486, 2010.

[86] S. Bala, M. Marcos, K. Kodys et al., "Up-regulation of MicroRNA-155 in macrophages contributes to increased tumor necrosis factor $\alpha(\mathrm{TNF} \alpha)$ production via increased mRNA half-life in alcoholic liver disease," Journal of Biological Chemistry, vol. 286, no. 2, pp. 1436-1444, 2011.

[87] M. V. S. Rajaram, B. Ni, J. D. Morris et al., "Mycobacterium tuberculosis lipomannan blocks TNF biosynthesis by regulating macrophage MAPK-activated protein kinase 2 (MK2) and microRNA miR-125b," Proceedings of the National Academy of Sciences of the United States of America, vol. 108, no. 42, pp. 17408-17413, 2011. 
[88] C. Cunha, C. Gomes, A. R. Vaz, and D. Brites, "Exploring new inflammatory biomarkers and pathways during LPS-induced M1 polarization," Mediators of Inflammation, vol. 2016, Article ID 6986175, 17 pages, 2016.

[89] Z.-X. Mo, J.-Q. Guo, D. She et al., "Infection by the nematode Angiostrongylus cantonensis induces differential expression of miRNAs in mouse brain," Journal of Microbiology, Immunology, and Infection, vol. 51, no. 1, pp. 94-102, 2018. 


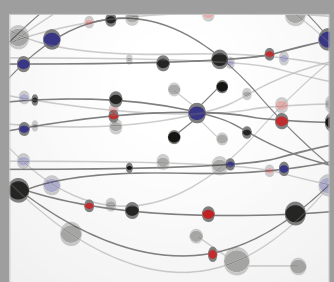

The Scientific World Journal
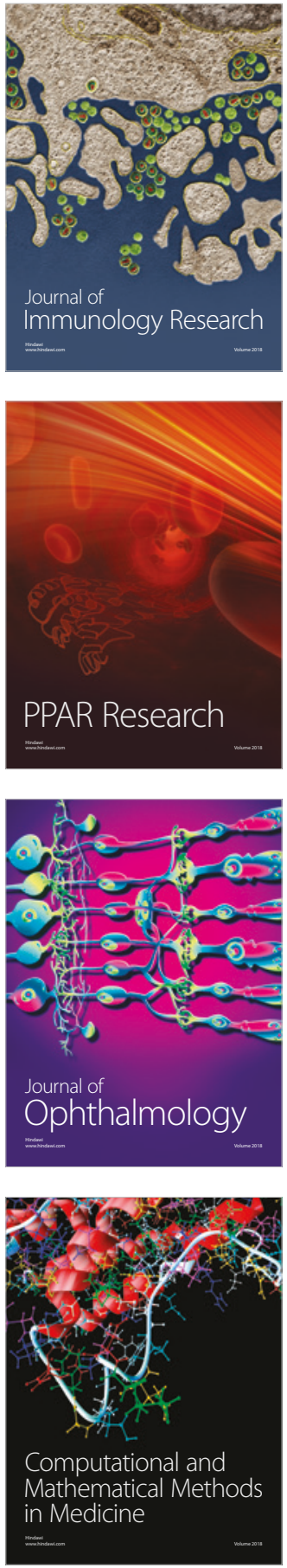

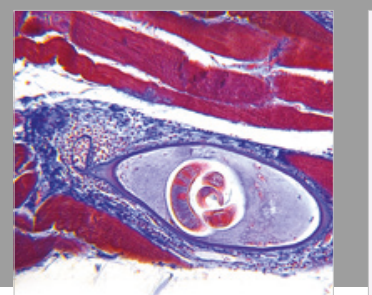

Gastroenterology Research and Practice

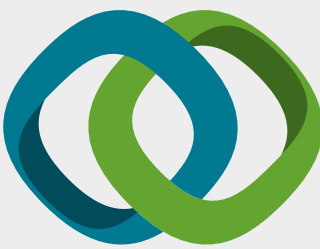

\section{Hindawi}

Submit your manuscripts at

www.hindawi.com
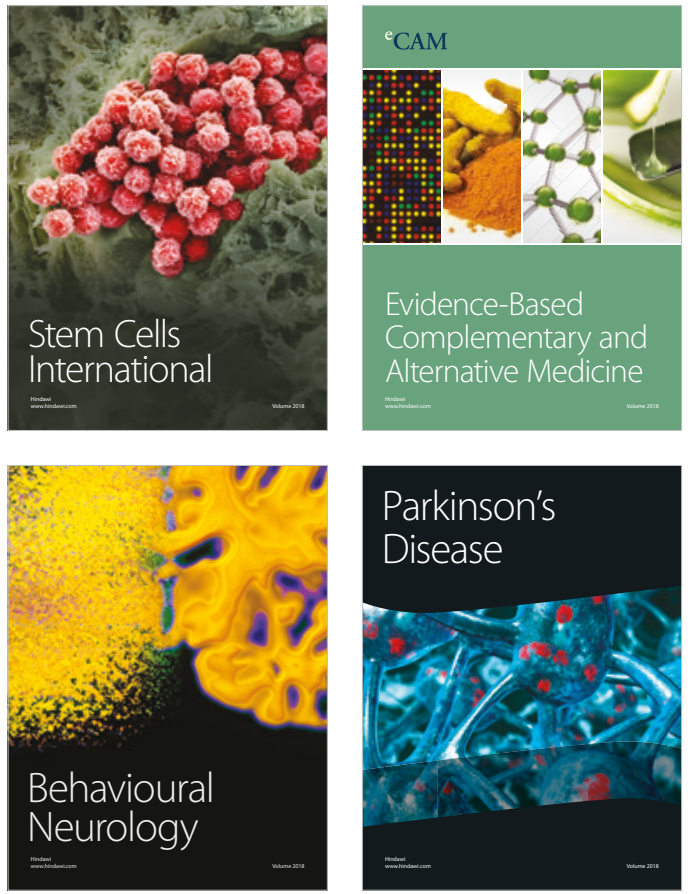

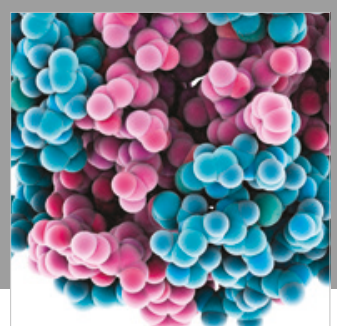

ournal of

Diabetes Research

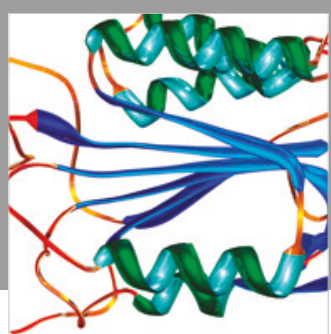

Disease Markers
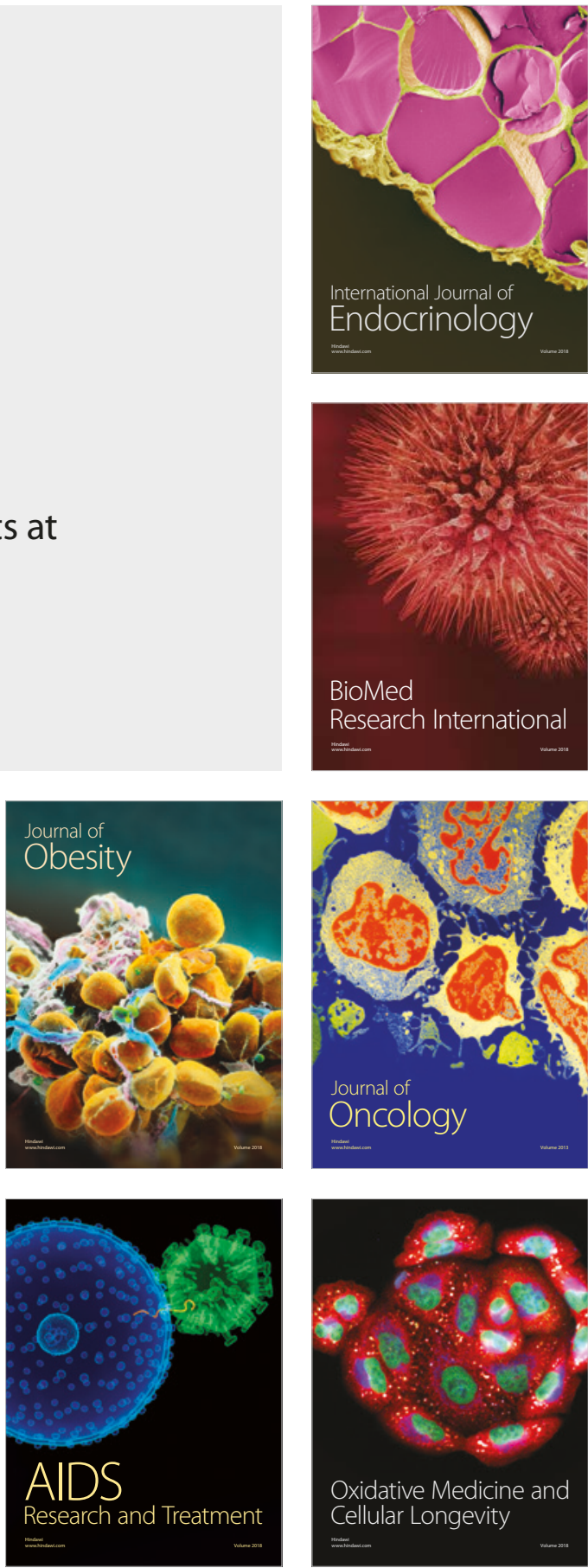